\title{
Water activity in Venus's uninhabitable clouds and other planetary atmospheres
}

\author{
John E. Hallsworth ${ }^{1 凶}$, Thomas Koop $\mathbb{D}^{2}$, Tiffany D. Dallas ${ }^{1}{ }^{1}$, María-Paz Zorzano ${ }^{3,4}$, \\ Juergen Burkhardt $\mathbb{1 0}^{5}$, Olga V. Golyshina ${ }^{6}$, Javier Martín-Torres ${ }^{4,7}$, Marcus K. Dymond ${ }^{8}{ }^{8}$, Philip Ball ${ }^{9}$ \\ and Christopher P. McKay ${ }^{10}$
}

\begin{abstract}
The recent suggestion of phosphine in Venus's atmosphere has regenerated interest in the idea of life in clouds. However, such analyses usually neglect the role of water activity, which is a measure of the relative availability of water, in habitability. Here we compute the water activity within the clouds of Venus and other Solar System planets from observations of temperature and water-vapour abundance. We find water-activity values of sulfuric acid droplets, which constitute the bulk of Venus's clouds, of $\leq \mathbf{0 . 0 0 4}$, two orders of magnitude below the 0.585 limit for known extremophiles. Considering other planets, ice formation on Mars imposes a water activity of $\leq \mathbf{0 . 5 3 7}$, slightly below the habitable range, whereas conditions are biologically permissive ( $>0.585$ ) at Jupiter's clouds (although other factors such as their composition may play a role in limiting their habitability). By way of comparison, Earth's troposphere conditions are, in general, biologically permissive, whereas the atmosphere becomes too dry for active life above the middle stratosphere. The approach used in the current study can also be applied to extrasolar planets.
\end{abstract}

T: here is currently a surge of interest in terrestrial aerobiology, and we now know that airborne microorganisms can be metabolically active $e^{1,2}$. As long as temperatures are biologically permissive, the abundance of liquid water within Earth's atmosphere favours physiological activity. The thermodynamic parameter water activity-the ratio between the water-vapour pressures of the solution and of pure water under the same conditions-is used to quantify the availability of water. This parameter applies to all solutions and phases-liquid, solid and gas. For planetary atmosphere applications, water activity is equivalent to relative humidity. The maximum possible value of equilibrium relative humidity is arbitrarily designated as $100 \%$, whereas the maximum possible water activity is attributed a value of 1 , for a given temperature and pressure combination. Water activity acts as a potent determinant of functionality for microbial cells ${ }^{3}$, so is also a key determinant of habitability ${ }^{4}$.

This raises the question of possible life in atmospheres beyond Earth. The atmospheres of other planetary bodies exhibit various combinations of temperature, pressure and relative humidityparameters that can be obtained through either calculations or direct measurements. Even if temperatures within the atmospheres of other planets permit the formation of liquid-water-containing droplets and may seem permissive for life as we know it, we must still ascertain the water activity of these droplets. This parameter is not only influenced by temperature and pressure but also by thermodynamic effects of ice and/or the presence of any solutes or co-solvent(s).

The recent suggestions of biogenic substances such as phosphine in the Venusian clouds ${ }^{5,6}$ is continuing a history of speculation about life on Venus ${ }^{7-9}$. Venus's surface is considered too hot for organic life forms but the lower cloud layer, at an altitude of $\sim 40$ to $70 \mathrm{~km}$, has a temperature range that makes it potentially habitable based on our knowledge of terrestrial-type life. Earlier studies have suggested the lack of liquid water, or at least the low availability of water, as a potential barrier to life ${ }^{10,11}$. Although several recent analyses have queried whether adequate water is available, they also propose active cellular metabolism in the sulfuric acid-rich droplets of the Venusian atmosphere ${ }^{5,12,13}$.

A thorough assessment of biophysical limits for life on Earth was carried out in the context of planetary protection by a 20132014 committee of the Mars Exploration Program Analysis Group (MEPAG) of NASA that aimed to identify 'special regions' of Mars ${ }^{4}$; that is, places that are biologically permissive for active terrestrial-type life. The MEPAG report identified that some metabolic processes occur down to $-40^{\circ} \mathrm{C}$, that is, below the recognized $\left(-18^{\circ} \mathrm{C}\right)$ limit for cell division, and stated that microbial metabolism and cell division had been documented only down to a water activity of 0.605 (the limit for cell division of the fungal xerophile Xeromyces bisporus). From a more recent study, we now know that metabolism, differentiation and cell division can occur down to a water activity of 0.585 (from a study of the fungal halophile/ xerophile Aspergillus penicillioides) ${ }^{14}$. We also know that microbes can remain dormant at water-activity values below their window for biotic activity, and then resume metabolism when water activity increases ${ }^{15}$. At the upper end of the water-activity scale, many microbes are active at a value of 1 (ref. ${ }^{16}$ ); and at high temperatures, microbial growth has been observed up to about $121^{\circ} \mathrm{C}$ (ref. ${ }^{4}$ ), but circumstantial evidence hints at possible metabolism close to $130^{\circ} \mathrm{C}$.

\footnotetext{
'Institute for Global Food Security, School of Biological Sciences, Queen's University Belfast, Belfast, UK. Faculty of Chemistry, Bielefeld University, Bielefeld, Germany. ${ }^{3}$ Centro de Astrobiologia (CSIC-INTA), Madrid, Spain. ${ }^{4}$ Department of Planetary Sciences, School of Geosciences, King's College, University of Aberdeen, Aberdeen, UK. ${ }^{5}$ Plant Nutrition Group, Institute of Crop Science and Resource Conservation, University of Bonn, Bonn, Germany. ${ }^{6}$ School of Natural Sciences and Centre for Environmental Biotechnology, Bangor University, Gwynedd, UK. 'Instituto Andaluz de Ciencias de la Tierra

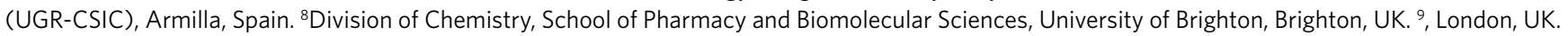
${ }^{10}$ Space Science Division, NASA Ames Research Center, Moffett Field, CA, USA. $\bigotimes_{e}$-mail: j.hallsworth@qub.ac.uk
} 


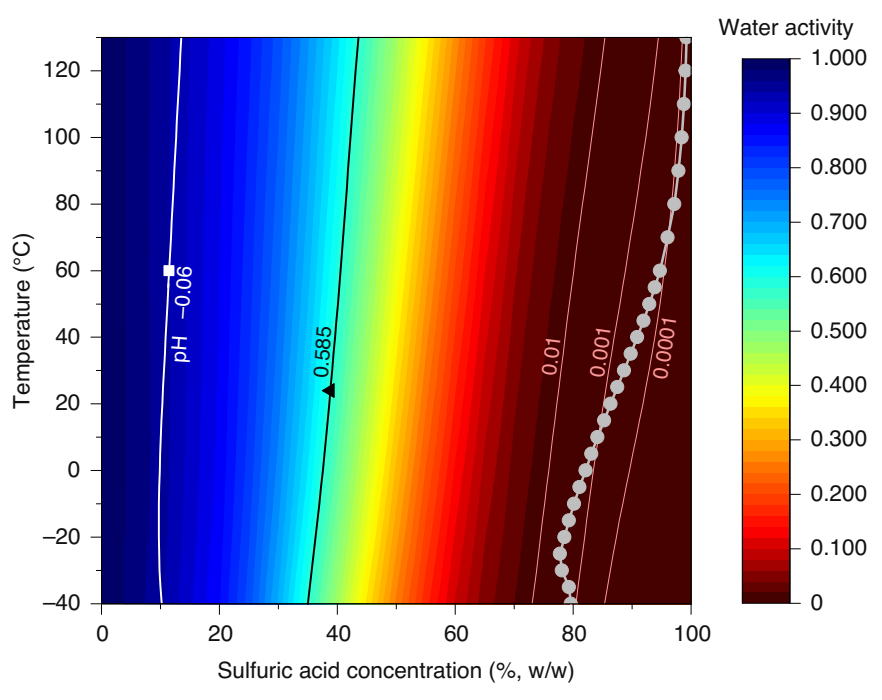

Fig. 1 | Map of water activity of liquid $\mathrm{H}_{2} \mathrm{SO}_{4}-\mathrm{H}_{2} \mathrm{O}$ mixtures as a function of temperature and sulfuric acid concentration over the temperature range pertinent to active life (between $-40^{\circ} \mathrm{C}$ and $130^{\circ} \mathrm{C}$ ). Values were calculated from the Gmitro and Vermeulen ${ }^{20,21}$ vapour-pressure data (Supplementary Table 1 and Methods). The colour scale indicates water-activity values from 0 to 1 . Several water activity isolines are shown: at a water activity of 0.585 (limit for active terrestrial life, which was observed at $24^{\circ} \mathrm{C}$ and pH 6.1 ref. ${ }^{14}$; black triangle) and extrapolated to other conditions (black isoline), and several isolines at very low water-activity values of $0.01,0.001$ and 0.0001 (thin rose-colour lines, right-hand side). The white square (left-hand side) indicates the tolerance limit for the most-extreme acidophile at an incubation temperature of $60^{\circ} \mathrm{C}$ and $\mathrm{pH}-0.06$ (ref. ${ }^{18}$; which is equivalent to $11.5 \%(\mathrm{w} / \mathrm{w}$ ) sulfuric acid at $60^{\circ} \mathrm{C}$ ); this $\mathrm{pH}$ limit was extrapolated to other conditions (white isoline). As no microbes are known to grow at 0.585 or $\mathrm{pH}-0.06$ at temperatures other than the original culture conditions (black triangle and white square, respectively), the assumption that life might be plausible under other conditions has not been substantiated, so each isoline acts as a conservative indicator of notional biophysical limits for life beyond that black triangle or white square. The grey circles show the conditions of Venus's clouds determined in this study; for details see main text and Table 1.

Here, in the light of this knowledge, including recent revisions onto our understanding of acidity and water-activity limits for activity of terrestrial microbes (see also below), we focus on Venus as a case study to quantify the water activity of clouds and determine whether terrestrial-type life is feasible there. However, the possibility of life in clouds can extend beyond Venus, so we also consider whether clouds on Jupiter and Mars have temperature and water-activity values consistent with habitability.

\section{Water activity and uninhabitability of Venus's clouds}

There is no a priori reason to suppose that putative Venusian life would have the same biochemical basis as that on Earth. In the absence of any concrete proposal for an alternative biochemistry, however, several studies have considered whether living systems comparable to those on Earth might find viable niches on Venus, suggesting that this might be possible within the droplets of sulfuric acid clouds ${ }^{5,12,13}$.

Concrete information about biophysical limits for cellular function comes primarily from laboratory-based studies of terrestrial extremophiles (see 'Acidity and water-activity limits for active life on Earth' and 'Determination of habitability for Venus's acid clouds' in Methods, and Supplementary text on biophysical limits of terrestrial microbes). These data indicate that microbial growth and metabolism cannot occur anywhere near the chemical conditions relevant for Venusian clouds, as revealed below (in this section). Much of the discussion of potential life in the atmosphere of Venus, both in the light of recent work ${ }^{5}$ and previous studies, focuses on the extreme acidity of sulfuric acid clouds ${ }^{12,13,17}$. This is not in itself an obvious obstacle because we know that some extreme acidophiles are capable of metabolism close to, and even below, $\mathrm{pH} 0$ (see references within ref. ${ }^{13}$ ). Indeed, it is sometimes assumed that the most acidophilic microbe can even grow in concentrated sulfuric acid. However, the record holder, the archaeon Picrophilus torridus, grows down to a $\mathrm{pH}$ of $-0.06\left(\text { at } 60^{\circ} \mathrm{C}\right)^{18}$, which is equivalent to only about $11.5 \%$ (w/w) sulfuric acid ${ }^{19}$.

Given that a permissive water activity is a prerequisite for active metabolism of terrestrial life forms, we considered the water activity within Venus's clouds. We determined how sulfuric acid modifies the water-vapour pressure of a liquid phase by converting water-vapour-pressure data for sulfuric acid-water $\left(\mathrm{H}_{2} \mathrm{SO}_{4}-\mathrm{H}_{2} \mathrm{O}\right)$ mixtures $^{20}$ to water-activity values (Fig. 1; data are provided in Supplementary Table 1). We observed a strong reduction of water activity even at modest sulfuric acid concentrations. We employed two independent thermodynamic models of $\mathrm{H}_{2} \mathrm{SO}_{4}-\mathrm{H}_{2} \mathrm{O}$ mixtures. The first model was from a study of Gmitro and Vermeulen ${ }^{20}$ and is particularly well suited for medium to high concentrations of (and up to pure) sulfuric acid over the entire temperature range of relevance to the current study. The second, the Extended Aerosol Inorganics Model (E-AIM) by Clegg et al. ${ }^{19}$ describes water activity and ion activities in multicomponent solutions, including $\mathrm{pH}$, and is applicable to sulfuric acid solutions of between 0 and $~ 80 \%$ (w/w) at temperatures from $<-73^{\circ} \mathrm{C}$ to $55^{\circ} \mathrm{C}$. For Fig. 1, water-activity values at temperatures that are pertinent to life (that is, those from -40 to $130^{\circ} \mathrm{C}$ ) were determined from the data presented in water-vapour-pressure tables, calculated by Gmitro and Vermeulen from their model ${ }^{20,21}$, at sulfuric acid concentrations between $10 \%$ and $100 \%(\mathrm{w} / \mathrm{w})$; intermediate values were then obtained by interpolation (Supplementary Table 1 and Methods).

Concentrations of 35.0, 37.5, 40.1 and $42.3 \%$ (w/w) sulfuric acid reduce water activity to the currently recognized (0.585) limit for life at $-40,0,50$ and $100^{\circ} \mathrm{C}(233,273,323$ and $373 \mathrm{~K})$, respectively (black isoline in Fig. 1). These sulfuric acid concentrations are consistent with the E-AIM ${ }^{19}$ for temperatures at $-40,0$ and $50^{\circ} \mathrm{C}(35.6$, 37.7 and $40.3 \%(\mathrm{w} / \mathrm{w}))$ and those published elsewhere for temperatures between 0 and $75^{\circ} \mathrm{C}(273 \text { and } 348 \mathrm{~K})^{22}$ (see Supplementary Fig. 1 and Supplementary Table $2 \mathrm{a}, \mathrm{b}$ for comparisons).

Here we derive the water-activity levels in Venus's clouds from direct observations and then determine the sulfuric acid concentration that corresponds to this water activity according to the solution chemistry. The relative humidity of the atmosphere can be calculated directly using observations of temperature, pressure and water-vapour mixing ratio ${ }^{23-26}$. At the altitudes pertinent to biology ( 40 to $70 \mathrm{~km}$; that is, about 130 to $-40{ }^{\circ} \mathrm{C}$ ), the relative humidity of the atmosphere varies yet remains less than $0.40 \%$ throughout this range. Because of the small droplet size, the water of the droplets and the ambient water vapour are assumed to be in equilibrium $^{27,28}$ (Methods); hence, these relative humidity values correspond to water activities in the droplets below 0.004 (that is, from 0.00003 to 0.0037; Table 1 and Fig. 2). Using data from Gmitro and Vermeulen ${ }^{20,21}$ (Methods), we observe that these water-activity values correspond to sulfuric acid concentrations of 77.8 to $99.2 \%$ $(\mathrm{w} / \mathrm{w})$ throughout the putative habitable zone, as indicated by the grey circles in Fig. 1 (see also Table 1). These concentrations are consistent with those of Clegg et al. ${ }^{19}$ in the temperature range -40 to $25^{\circ} \mathrm{C}$ (even though they lie beyond the stated validity range of the E-AIM). Our results for water activity are consistent with published observations and published model calculations of acid content in Venus's clouds ${ }^{29-31}$. Given that Earth's entire functional biosphere spans only about 0.415 water-activity units, from 1 to 0.585 (ref. ${ }^{32}$ ), 
Table 1 | Water activity and sulfuric acid concentrations of droplets in the Venusian cloud layer ${ }^{\mathrm{a}}$

\begin{tabular}{|c|c|c|c|c|c|}
\hline Temperature $\left({ }^{\circ} \mathrm{C}\right)^{\mathrm{b}}$ & $\begin{array}{l}\text { Altitude above mean } \\
\text { ground level }(\mathrm{km})^{\mathrm{b}}\end{array}$ & Pressure (bar) ${ }^{b}$ & $\begin{array}{l}\text { Relative humidity } \\
(\%)^{c}\end{array}$ & $\begin{array}{l}\text { Water activity }{ }^{d} \text { of cloud } \\
\text { droplets }\end{array}$ & $\begin{array}{l}\text { Sulfuric acid concentration of } \\
\text { cloud droplets }(\%, w / w)^{\text {e }}\end{array}$ \\
\hline-35 & 67.02 & 0.067 & 0.1803 & 0.001803 & 79.3 \\
\hline-30 & 65.02 & 0.097 & 0.3051 & 0.003051 & 78.1 \\
\hline-25 & 63.40 & 0.131 & 0.3692 & 0.003692 & 77.8 \\
\hline-20 & 62.30 & 0.158 & 0.3235 & 0.003235 & 78.6 \\
\hline-10 & 59.94 & 0.238 & 0.2440 & 0.002440 & 80.2 \\
\hline-5 & 59.09 & 0.276 & 0.1982 & 0.001982 & 81.1 \\
\hline 0 & 58.32 & 0.313 & 0.1605 & 0.001605 & 82.1 \\
\hline 5 & 57.60 & 0.354 & 0.1296 & 0.001296 & 83.1 \\
\hline 10 & 56.93 & 0.394 & 0.1043 & 0.001043 & 84.2 \\
\hline 30 & 54.92 & 0.538 & 0.0430 & 0.000430 & 88.7 \\
\hline 35 & 54.44 & 0.579 & 0.0351 & 0.000351 & 89.8 \\
\hline 40 & 53.97 & 0.619 & 0.0288 & 0.000288 & 90.9 \\
\hline 45 & 53.48 & 0.666 & 0.0239 & 0.000239 & 91.9 \\
\hline 50 & 52.99 & 0.712 & 0.0199 & 0.000199 & 92.9 \\
\hline 55 & 52.50 & 0.764 & 0.0167 & 0.000167 & 93.9 \\
\hline 60 & 52.02 & 0.815 & 0.0140 & 0.000140 & 94.7 \\
\hline 70 & 50.87 & 0.952 & 0.0104 & 0.000104 & 96.1 \\
\hline 80 & 49.67 & 1.061 & 0.0075 & 0.000075 & 97.2 \\
\hline
\end{tabular}

aBased on the assumptions that droplets are in equilibrium with the atmospheric relative humidity and that the primary sulfate species is sulfuric acid. ${ }^{\mathrm{b}} \mathrm{Altitude}$, temperature and pressure values are from entry-probe data with uncertainties of $\pm 1 \mathrm{~km}, \pm 1 \mathrm{~K}$ and $\pm 5 \%$, respectively ${ }^{23}$. ${ }^{c}$ Values were computed using the mixing ratio of water from observations as parameterized by Gao et al. ${ }^{26}$. Uncertainty, based on reported uncertainties in atmospheric profile, is $\pm 30 \%$ of the value. dDerived by dividing relative humidity by 100 , based on the assumption that droplets are in equilibrium with the atmospheric relative humidity. Each value is pertinent to the stated temperature (column 1). Uncertainty, based on reported uncertainties in atmospheric profile, is $\pm 30 \%$ of the value. ${ }^{\mathrm{e} V a l u e s, ~ s t a t e d ~ t o ~ o n e ~ d e c i m a l ~ p l a c e, ~ w e r e ~}$ derived from interpolations of the data of Gmitro and Vermeulen ${ }^{20,21}$ to the water-activity values in column 5 , based on the assumption that the primary sulfate species is sulfuric acid (Methods).

the thermodynamic distance between the 0.585 water-activity limit and the water activity of Venus's cloud droplets seems unbridgeable (Fig. 1). In other words, there is an enormous distance on the water-activity scale between the limits for metabolism of terrestrial extremophiles and the conditions of the Venusian cloud layer (Fig. 3). Furthermore, terrestrial life cannot survive extreme acid concentrations equivalent to those found in the Venusian clouds (see below; Fig. 3).

For cloud droplets to be habitable, their water activity would have to be strongly out of equilibrium, meaning that the water activity is not determined by ambient relative humidity. Indeed, their water activity would have to be enhanced by a factor of about 150 with respect to the ambient water vapour. In general, authors of different studies (ourselves included) agree that the droplets making up Venus's clouds are in equilibrium with the atmosphere; both data and theoretical evidence support this view (Supplementary text relating to the equilibration of droplets) ${ }^{5,29}$.

Note that even at temperatures below $0{ }^{\circ} \mathrm{C}$, no ice can form in the cloud layer at such low water-activity values. In the binary-phase diagram of sulfuric acid and water ${ }^{33}$, several crystalline sulfuric acid hydrates are stable at lower temperatures, but at the high acid concentrations considered here (of $>78 \%(w / w)$ ), in principle only the sulfuric acid monohydrate or pure crystalline sulfuric acid can form. However, laboratory experiments have shown that neither of these phases crystallizes readily, even in bulk samples, and that they instead form metastable-solution droplets ${ }^{33}$. Furthermore, observations in Earth's stratospheric aerosol layer support this notion ${ }^{30}$.

Seager et al. ${ }^{13}$ speculated that Venusian microbes would have adaptations to capture and retain water, but we have yet to identify any terrestrial microbe able to obtain and accumulate water from the vapour phase at $<0.40 \%$ relative humidity ${ }^{4}$. Furthermore, we believe that under the hostile conditions in the Venusian atmosphere any cells would probably perish (even dormant cells); see 'Acidity and water-activity limits for active life on Earth' in Methods. The water-activity limits for active life are determined by thermodynamics and the need for water as the biophilic solvent for complex macromolecules ${ }^{34}$. At low water activity, microbial cells adapt to retain their functionality by changing the composition of the plasma membrane; by accumulating stress metabolites ${ }^{35}$; by preferentially accumulating chaotropic substances at low temperatures 


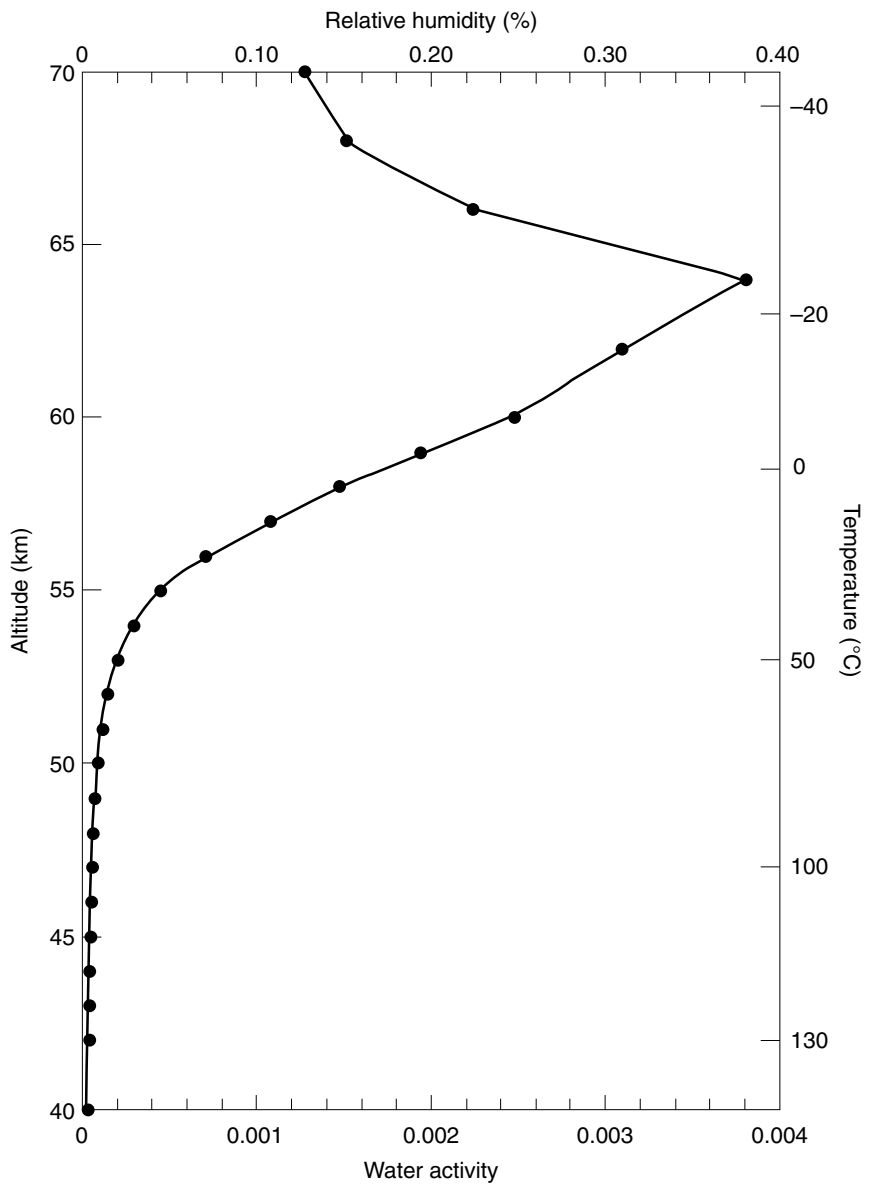

Fig. 2 | Water activity and relative humidity of the Venusian atmosphere in the region where temperatures are in the range of possible biological interest (between $-40^{\circ} \mathrm{C}$ and $130^{\circ} \mathrm{C}$ ). The uncertainty in altitude is smaller than the size of the data-point markers. The uncertainty in water activity is $\pm 30 \%$ of the value, as described in the main text.

to retain flexibility of their macromolecular systems ${ }^{36}$; and in other ways. Such adaptations mitigate against stresses induced by low water activity, osmotic stress, and other biophysical activities of solutes but this mitigation only extends the windows for cellular functionality within finite limits and cannot circumvent the need for a biologically permissive water activity ${ }^{36-38}$.

In their analysis of cloud habitability, Seager et al. ${ }^{13}$ state that cells can be destroyed due to chemical modifications to metabolites and cellular macromolecules (these are detailed in our Fig. 3). This is consistent with studies showing that extremely acidic solutions rapidly kill the cells of many microbes at $\mathrm{pH}<1$ (ref. ${ }^{39}$ ). However, we argue that the problems go far deeper. We can see that sulfuric acid, at concentrations thought relevant to Venusian cloud droplets, reduces water activity far below a level where the water can function as a biophilic solvent for complex macromolecules (Table 1 and Figs. 1 and 3). Sulfuric acid dehydrates the cellular systems, removes water from biomacromolecules, reduces hydrophobic interactions and damages plasma-membrane integrity (Fig. 3). For both the polyextremophile Acidihalobacter aeolianus ('Acidity and water-activity limits for active life on Earth' in Methods) and the thermoacidophile $P$. torridus, sulfuric acid tolerance limits for growth are an order of magnitude lower than those found in the clouds of Venus (Fig. 3).

It is important to remember what the hydration shell of a protein actually does for functionality. There is evidence that the surface of a typical protein (lysozyme, for example) must have at least $50 \%$ water coverage to be functional ${ }^{40}$. This is thought to correspond to $66 \%$ of the purely hydrophilic regions, and to coincide with a percolation threshold in two dimensions. Of course, one cannot assume that macromolecules in a Venusian organism would share the same features as those of terrestrial organisms, but the real point is what role this water coverage plays. The dynamics of proteins and their hydration spheres are closely coupled, and it is thought that fluctuations of the solvent (due to spontaneous rearrangements of the hydrogen-bonded network) are needed to 'awaken' those in the protein and give it the plasticity required for functionality ${ }^{41}$. For additional information, see 'Determination of habitability for Venus's acid clouds' in Methods and Supplementary text for Fig. 3.

\section{Analyses for clouds of Jupiter, Mars, Earth and exoplanets}

Determinations of water activity, as an important first step in assessing habitability of clouds, can also be made for Jupiter, Mars, Earth and exoplanets. For Jupiter, there has been only one entry probe and it appears to have entered into an unusually dry region of the Jovian atmosphere. Furthermore, dry and wet regions are present in a complex pattern of local meteorolog $y^{42}$. Here we use the temperature-pressure profiles from this probe, the Galileo Atmospheric Entry Probe ${ }^{43}$. The water-vapour mixing ratio, that is, the molar ratio of water gas to all other gases present, is not well constrained. The Galileo Atmospheric Entry Probe measured water-vapour mixing ratios of $(4.7 \pm 1.5) \times 10^{-5}$ at $11-11.7$ bar and $(4.9 \pm 1.6) \times 10^{-4}$ at $17.6-20.9$ bar $\left(\right.$ ref. $\left.^{44}\right)$. These values are about $3 \%$ and $30 \%$, respectively, of the water-vapour mixing ratios expected if water on Jupiter had the same relative abundance of oxygen and hydrogen as the Sun. Conversely, Li et al. ${ }^{45}$ used the results from the Juno mission, which orbited Jupiter, to infer that the water-vapour mixing ratio in the range of approximately 0.7 to 30 bar is about 2.7 times the value expected for solar abundances. Although it is clear that the water-vapour mixing ratio is variable and the measurements uncertain, the value set by solar abundances deep in the Jovian atmosphere is plausible. This value is $1.71 \times 10^{-3}$ (ref. ${ }^{46}$ ).

Higher in Jupiter's atmosphere, the water-vapour mixing ratio is likely to be reduced due to the removal of water by condensation. However, in the region of interest between 0.1 bar and 20 bar, the atmospheric temperature profile decreases smoothly with decreasing pressure so there is no temperature minimum to act as a cold trap $^{43}$. Thus, a uniform mixing ratio of water vapour throughout this region is plausible (see, for example, ref. ${ }^{46}$ ). If anything, this assumption will overestimate the water activity at lower pressures on Jupiter. Figure 4 shows water activity in the Jovian atmosphere as a function of temperature, from $-50^{\circ} \mathrm{C}$ to $70^{\circ} \mathrm{C}$. Condensation occurs at about $10^{\circ} \mathrm{C}$ (at 5.5 bar) and becomes ice below $0{ }^{\circ} \mathrm{C}$ (strictly, there is also an effect of pressure on water and ice activity, but this is probably negligible given the other uncertainties). With further decreases in temperature, the water activity of ice decreases. In this analysis, we have neglected the effects of ammonia $\left(\mathrm{NH}_{3}\right)$ or other atmospheric components on the water activity of liquid water or on the freezing-point depression of the ice. These effects become more important near the ammonia clouds found at higher elevations (lower pressures and lower temperatures) that are not pertinent to the current analysis. The water activity is suitable for life $(>0.585)$ for temperatures between approximately $10^{\circ} \mathrm{C}$ and $-40^{\circ} \mathrm{C}$. According to these parameters, the atmosphere of Jupiter may therefore be more suitable for hosting terrestrial-type life than that of Venus (but whether it could serve as a suitable location for initiating life is another matter).

For Mars, even a casual inspection of the in situ conditions reveals that the clouds are not biologically permissive due to the low temperatures that are inconsistent with cellular function (there is also high ultraviolet radiation that can be lethal for atmospheric microbes ${ }^{47}$ ). Whereas clouds have been observed in the atmosphere 


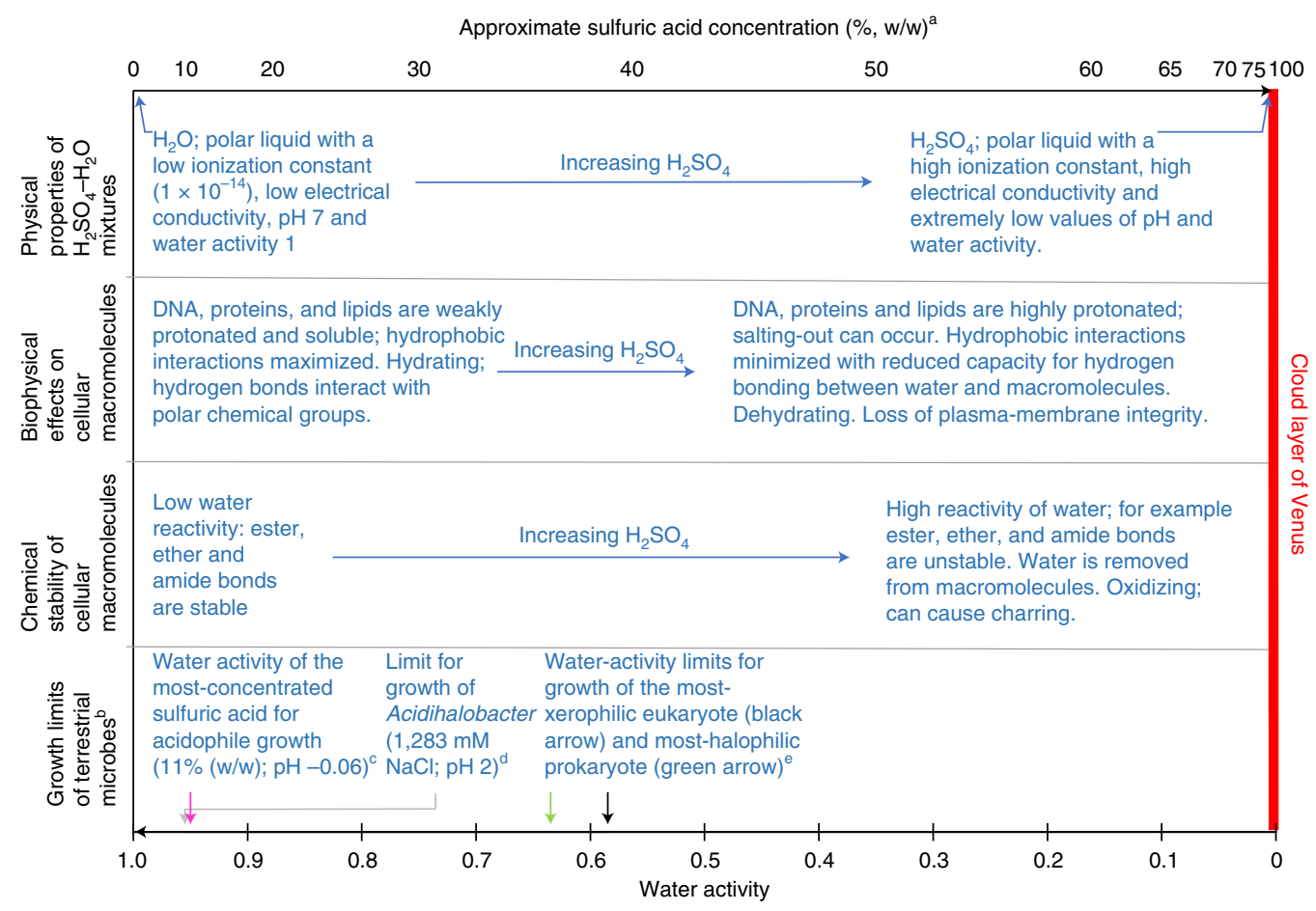

Fig. 3 | Schematic showing the implications of the water-activity values of $\mathrm{H}_{2} \mathrm{SO}_{4}-\mathrm{H}_{2} \mathrm{O}$ mixtures, including those found within the cloud layer of Venus, for cellular terrestrial-type life. The narrow red zone (which looks like a thick red line) indicates the water activity of the $\mathrm{H}_{2} \mathrm{SO}_{4}-\mathrm{H}_{2} \mathrm{O}$ droplets of $\mathrm{Venus's}$ lower cloud layer (altitude about 40 to $70 \mathrm{~km}$ ) within the temperature range that is consistent with habitability, based on knowledge of Earth's microbial

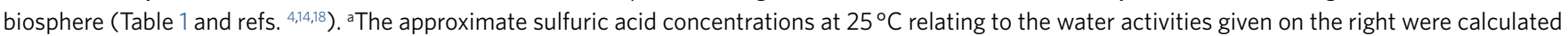
using the $\mathrm{E}_{-} \mathrm{AIM}^{19}$, and Gmitro and Vermeulen ${ }^{20,21}$, at $25^{\circ} \mathrm{C}$ and the average concentration is given here (Methods). ${ }^{\mathrm{b}}$ For details, see main text. ${ }^{\mathrm{C}} \mathrm{The}$ thermoacidophile, P. torridus, is not known to grow at lower water activity than that of $11.5 \%$ (w/w) sulfuric acid (about 0.950 ${ }^{19}$, pink arrow; Methods). For further information about $\mathrm{H}_{2} \mathrm{SO}_{4}-\mathrm{H}_{2} \mathrm{O}$ mixtures, see Supplementary text for Fig. 3. ${ }^{\mathrm{d}}$ This polyextremophile (A. aeolianus) is halotolerant and acidophilic but grows only down to $\mathrm{pH} 2$ at high $\mathrm{NaCl}$ at $30^{\circ} \mathrm{C}$, with a culture-medium water activity of 0.955 (grey arrow; see also main text). ${ }^{\mathrm{e}} \mathrm{These}$ are two haloarchaea able to grow down to 0.635 water activity (green arrow) at high salt concentrations ${ }^{3}$ and the acidotolerant xerophile/halophile $A$. penicillioides can grow down to 0.585 water activity (black arrow) at high glycerol concentration ${ }^{14}$. These microbes cannot tolerate high sulfuric acid concentrations but are shown here as they represent the water-activity limits for active prokaryote and eukaryote life, respectively (note that at $24^{\circ} \mathrm{C}, 0.585$ water activity is equivalent to $39.0 \%(\mathrm{w} / \mathrm{w})$ sulfuric acid $(\mathrm{pH}-1.55)$ according to Clegg et al. $\left.{ }^{19}\right)$.

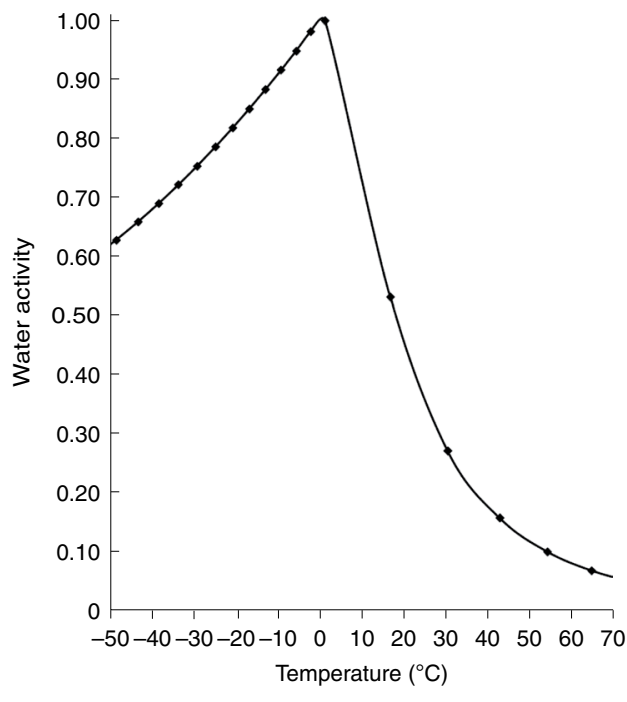

Fig. 4 | Water activity in the atmosphere of Jupiter over the altitude range that may be suitable for life. These temperatures correspond to pressures of 2.5 to 10 bar. Temperature and pressure are taken from the Galileo Atmospheric Entry Probe data ${ }^{23}$. A constant water-vapour mixing ratio is assumed to be set by the solar ratio of $\mathrm{O} / \mathrm{H}^{46}$. The water activity decreases for temperatures less than $0^{\circ} \mathrm{C}$ due to the reduction in water activity of ice with temperature. of Mars, the temperatures are less than $-73^{\circ} \mathrm{C}(200 \mathrm{~K})$ regardless of altitude or location as determined by entry probes and global remote sensing ${ }^{48,49}$. Therefore, at best, there are ice clouds, and microbial cells are not known to be able to access water at these extremely low temperatures. Furthermore, the water activity of ice at $-73^{\circ} \mathrm{C}$ is about 0.537 , and then drops sharply with decreasing temperature (computed from vapour-pressure formulae of liquid water and ice in ref. ${ }^{50}$ ), so the water activity is also below the limit for active terrestrial-type life.

For Earth, typical water-mixing ratio profiles show that the upper stratosphere and mesosphere are too dry to be permissive for active life, while in the troposphere water activity is very variable spanning the entire range between 0 and 1, depending on location, season and daily weather (Figs. 5 and 6; see Methods for details). Nearly all clouds in Earth's atmosphere are composed of either liquid water or water ice; the water activity of liquid-water droplets is practically 1 (see Supplementary text for Fig. 6b) so is permissive for active life. The water activity of ice clouds, such as tropospheric cirrus clouds, depends on temperature and is in theory biologically permissive down to $-58^{\circ} \mathrm{C}(215 \mathrm{~K})$. We note that temperatures below this $-58^{\circ} \mathrm{C}$ limit typically occur only in the upper troposphere, polar stratosphere, and in the mesosphere. Our analysis suggests that the vast majority of clouds in the troposphere are above the water-activity limit of 0.585 and, thus, consistent with active life.

Our approach of using temperature, pressure and water-vapour mixing ratio profiles to determine water activity can be applied to exoplanets. In principle, values for these three parameters can be 


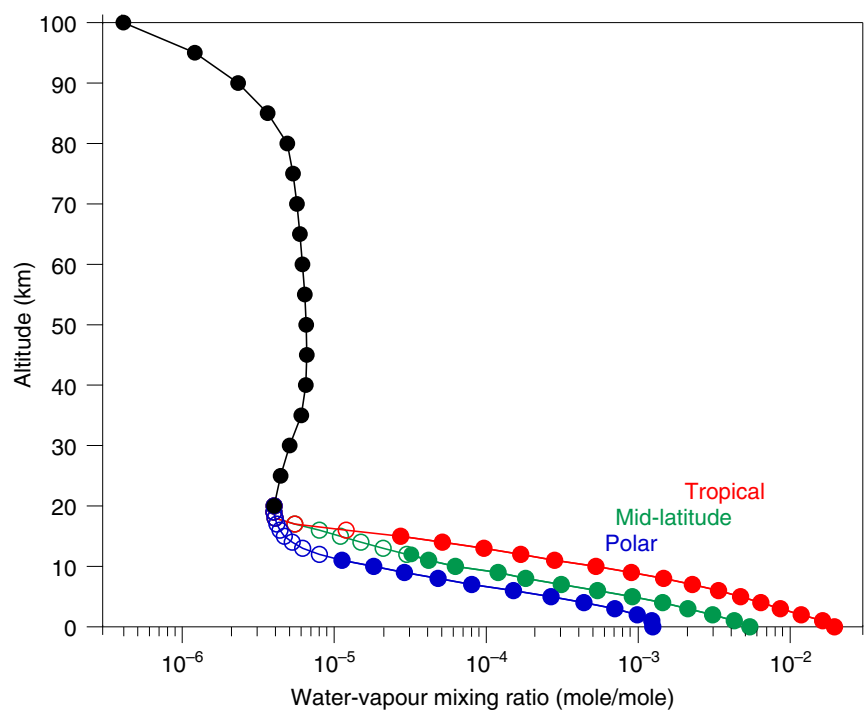

Fig. 5 | Average water-vapour mixing ratio profiles in Earth's atmosphere under cloud-free conditions. At low altitude, three different profiles represent the polar, mid-latitude and tropical troposphere ${ }^{91}$ (solid blue, green and red circles, respectively). These profiles were then interpolated (open circles) to the average profile in the stratosphere and mesosphere ${ }^{92}$ (solid black circles; see Methods).

calculated from transit measurements as an exoplanet moves in front of its star. Considering the problem in general, we can work out the case for an exoplanet atmosphere assuming that the water-vapour mixing ratio is set by the solar ratio of $\mathrm{O} / \mathrm{H}$. For example, Kreidberg et al. $^{51}$ found the water-vapour abundance of a Jupiter-sized exoplanet to be equal to the value for the Sun within the measurement uncertainties. Figure 7 shows a generalized water-activity analysis for exoplanets: the curve of pressure and temperature corresponds to the water activity currently regarded as the minimum for active life (0.585) (see also Supplementary text for Fig. 3). Profiles to the right of this curve will have a lower water activity, whereas profiles to the left will have a higher water activity. Ice clouds will have a lower water activity set by their temperature. The lowest temperature considered here is $-59^{\circ} \mathrm{C}$ because below that value the water activity of ice is less than 0.585 .

\section{Implications and perspectives}

For a desiccated environment, whether a planetary atmosphere, surface or subsurface, the presence of liquid water does not necessarily indicate habitability. Temperature must be permissive for cellular integrity and function, energy sources and nutrients must be available and, critically, water activity must also be permissive for life. This is illustrated by our case study of Venus's clouds where viable microbe-water relations are a key prerequisite that - based on our knowledge of life on Earth-cannot be ignored. Indeed, it is only the two parameters of temperature and water activity that are considered determinants of habitability for the purposes of planetary protection ${ }^{4}$. It has been suggested that the droplets of Venus's clouds can act as a protective environment for microorganisms ${ }^{13}$, but we believe that cells could not retain their integrity and/or functionality there due to the low water activity and biophysical and chemical effects of the highly concentrated sulfuric acid. Whereas we find the Greaves et al..$^{5}$ report of phosphine to be highly intriguing, other studies refute this finding, for example, refs. ${ }^{52-54}$. On the basis of the current study, we must imagine a qualitatively new type of organism to invoke a plausible story about life in the atmosphere of Venus, at least for life as we know it.
We found that, in relation to water activity and temperature, the in situ conditions make Jupiter's clouds the most likely cloud formations in which life could exist in our Solar System apart from those of Earth. A similar analysis would be of interest for Saturn, Uranus or Neptune, once entry-probe missions have been conducted. At present, we lack empirical data for suitable exoplanet atmospheres. However, we mapped out an approach whereby water-activity determinations can be made. It may be that such analyses form a key part of assessments in future to identify exoplanets based on determinations of their habitability. The James Webb Space Telescope (JWST) will be able to determine atmospheric profiles of temperature, pressure and water abundance in exoplanet atmospheres ${ }^{55}$, and these will allow assessments of water activity in their atmospheres using our approach.

On Earth, life in the atmosphere has co-evolved with life in the oceans, on the surface and in the subsurface. Terrestrial microorganisms are known to influence the atmospheric composition, hydrological cycle and meteorology. For example, microbes produce and consume various greenhouses gases; synthesize the stress metabolite dimethyl sulfide that volatilizes and influences climate $^{56}$; nucleate ice ${ }^{57}$; and drive cloud formation and precipitation. According to the findings of the current study, Earth's troposphere is for the most part biologically permissive, but the middle- and upper atmosphere becomes too dry for active life. Follow-on studies are also needed to consider the type of intimate relationship that can occur between the atmosphere's microbiome and other aspects of the planetary atmosphere, including climate and weather.

It should also be noted that on Earth (and to a lesser extent on Jupiter and possibly on Venus), atmospheric conditions can be dynamic. Therefore, the calculations presented here should be considered as representative rather than fixed. This said, the temperature, pressure and composition can be determined by direct measurements of planetary atmospheres (from probes or by
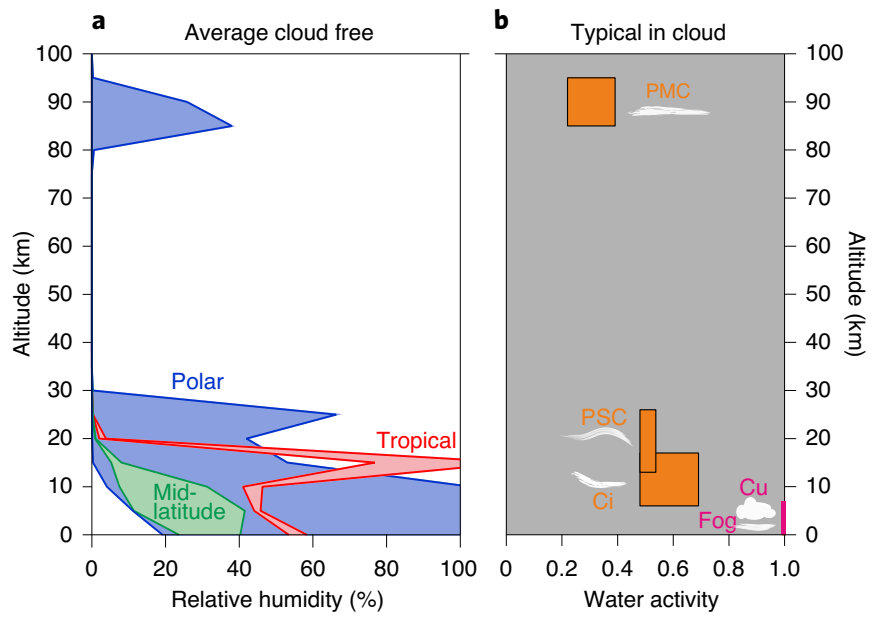

Fig. 6 | Relative humidity and water-activity ranges at different altitudes in Earth's atmosphere for cloud-free conditions and in clouds.

a, Maximum and minimum relative humidity averages for January and July reference atmosphere for cloud-free conditions in polar, mid-latitude and tropical regions (blue, green and red, respectively), calculated using temperature-pressure data from the COSPAR International Reference Atmosphere ${ }^{90}$ and the water-vapour mixing ratio profiles from Fig. 5 (Methods). b, Typical in-cloud water-activity values. Liquid-water clouds, for example, fog and cumulus clouds (Cu), are indicated by the magenta bar (bottom right). Ice clouds, for example, cirrus clouds (Ci), type-II polar stratospheric clouds (PSC) and polar mesospheric clouds (PMC), are indicated as orange boxes. For further information on cloud types, conditions for formation and location, see Supplementary Table 3. Between about 26 and $85 \mathrm{~km}$, it is too dry for clouds to form and persist. 


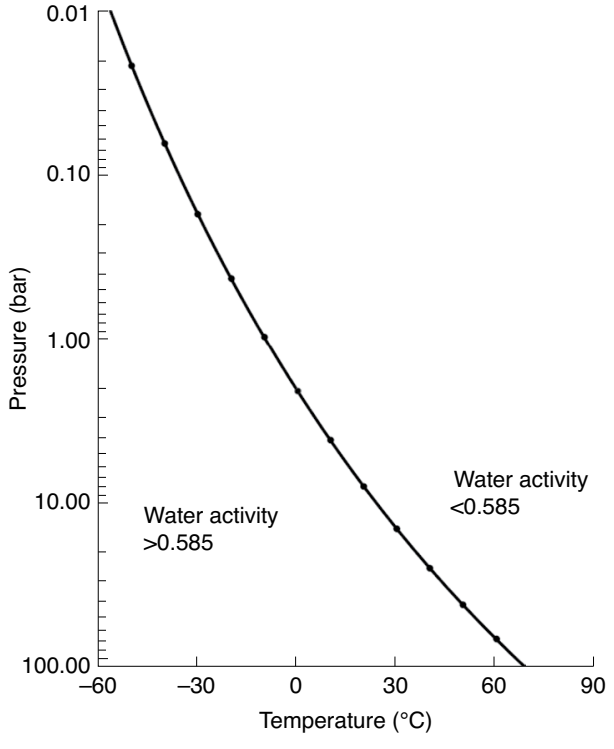

Fig. 7 | Generalized water-activity analysis for an exoplanet atmosphere. Temperature and pressure profile that corresponds to a water activity of 0.585-the limit for active life $\mathrm{e}^{14}$-for a water-vapour mixing ratio set by the solar ratio of $\mathrm{O} / \mathrm{H}$. For values to the right of the curve, the values of water activity are less than the 0.585 water-activity limit whereas for values to the left of the curve, the water activity is greater than this limit. The lowest temperature considered is $-59^{\circ} \mathrm{C}$; below that value, the water activity of ice is less than 0.585 .

remote-sensing methods) and these data provide a way to assess water activity. This methodology has planetary protection implications in relation to the potential designation of planetary atmospheres as 'special regions'. We believe that the quantitative tools developed here can also be used to determine the water activity in exoplanet atmospheres thereby narrowing the search for life within our Solar System and beyond.

\section{Methods}

Water activity of $\mathrm{H}_{2} \mathrm{SO}_{4}-\mathrm{H}_{2} \mathrm{O}$ mixtures. All solutes/co-solvents depress the water activity of solute- or co-solvent-water mixtures. We sought to identify datasets and models that enable quantification of water activity for $\mathrm{H}_{2} \mathrm{SO}_{4}-\mathrm{H}_{2} \mathrm{O}$ mixtures over a range of temperatures and sulfuric acid concentrations. For this purpose, we used two independent, semi-empirical thermodynamic models. Gmitro and Vermeulen ${ }^{20,21}$ used experimental data to provide a comprehensive model dataset for vapour pressures of water, sulfuric acid and sulfur trioxide for aqueous sulfuric acid solutions from 10 to $100 \%\left(\mathrm{w} / \mathrm{w}\right.$ ) at temperatures from -50 to $400^{\circ} \mathrm{C}$. These were tabulated in a supplement to the paper ${ }^{20,21}$, and we extracted the water-vapour pressures from -40 to $130^{\circ} \mathrm{C}$. These data were then converted to water activity by dividing them by the water-vapour pressure over pure water at the same temperature (the values for pure water were obtained from Murphy and Koop ${ }^{50}$ (up to and including $0^{\circ} \mathrm{C}$ ) and Wagner and Pruss ${ }^{58}$ (higher than $0{ }^{\circ} \mathrm{C}$ ).

These water-activity values were used in the current study to produce Fig. 1 by interpolation using the contour plot function of OriginPro 2021 (version 9.8.0.200). We also present four isolines of constant water activity in Fig. 1. For the 0.585 water-activity isoline, the corresponding sulfuric acid concentrations were obtained by linear interpolation between the nearest water activity: sulfuric acid concentration data pairs from Supplementary Table 1. For the $0.01,0.001$ and 0.0001 water-activity isolines, linear interpolation between the nearest $\log$ (water activity):sulfuric acid concentration data pairs were used because water activtiy changes by several orders of magnitude in that concentration range. The Gmitro and Vermeulen ${ }^{20,21}$ data were also used to calculate interpolated sulfuric acid concentrations in Venus cloud droplets from water activities (see below and Table 1), again by linear interpolation between the nearest $\log$ (water activity):sulfuric acid concentration data pairs.

The E-AIM ${ }^{19}$ was employed to calculate water activities at predefined sulfuric acid concentrations and vice versa, as well as corresponding $\mathrm{pH}$ values. For that purpose, we employed model I (either option 1, 'simple', or option 3, 'aqueous solution') of the online version of the E-AIM ${ }^{59}$. The E-AIM is valid from -93.2 to $56.9^{\circ} \mathrm{C}(180$ to $330 \mathrm{~K})$ and up to sulfuric acid concentrations of about $80 \%(\mathrm{w} / \mathrm{w})$.
For the $\mathrm{pH}$ calculations, the E-AIM provides the mole-fraction-based $\mathrm{H}^{+}$activity coefficient, which was converted to the molality-based activity coefficient ${ }^{60}$ and then the molality-based $\mathrm{H}^{+}$activity in solution, from which the molality-based $\mathrm{pH}$ (the negative decadal logarithm of $\mathrm{H}^{+}$activity according to International Union of Pure and Applied Chemistry (IUPAC) convention ${ }^{61}$ ) is derived. The E-AIM ${ }^{19}$ was used to calculate the $\mathrm{pH}-0.06$ isoline shown in Fig. 1 for temperatures between $-40^{\circ} \mathrm{C}$ and $56^{\circ} \mathrm{C}$. Between $25^{\circ} \mathrm{C}$ and $56^{\circ} \mathrm{C}$, there was a perfectly linear behaviour for corresponding sulfuric acid concentrations, which enabled a linear extrapolation to $130^{\circ} \mathrm{C}$. The E-AIM ${ }^{19}$ was also used to calculate sulfuric acid concentrations in Venus's cloud droplets from water activities, for comparison with those calculated from the Gmitro and Vermeulen ${ }^{20,21}$ data. Although the agreement is very good for temperatures up to about $25^{\circ} \mathrm{C}$, we note that all these values are outside the stated validity range of the E-AIM ${ }^{19}$ (sulfuric acid concentrations less than $\sim 80 \%(\mathrm{w} / \mathrm{w})$ and water activity higher than 0.01$)$. See also Supplementary text relating to validation of water activity for $\mathrm{H}_{2} \mathrm{SO}_{4}-\mathrm{H}_{2} \mathrm{O}$ mixtures.

Relative humidity and water activity of Venus's atmosphere. Small droplets within clouds rapidly equilibrate with relative humidity (as shown in this section, below), so it was imperative to quantify relative humidities of the Venusian atmosphere for the altitude/temperature range that is potentially habitable according to our knowledge of life on Earth. Between about 40 and $70 \mathrm{~km}$ altitude, the atmosphere of Venus has temperatures and pressures similar to those of the lower atmosphere of Earth. To compute the physical properties in this region for Venus, we used the direct measurements of pressure and temperature from entry probes for equatorial latitudes, $\pm 30^{\circ}\left(\right.$ ref. ${ }^{23}$ ). These datasets report values for every kilometre in altitude and the reported uncertainties in this profile are $\pm 0.15 \mathrm{~km}$ for altitude, $\pm 5 \mathrm{~K}$ for temperature and $\pm 5 \%$ for pressure ${ }^{23}$. Day-to-night temperature differences are only about $\pm 5 \mathrm{~K}$ (ref. ${ }^{23}$ ). The water-vapour mixing ratio is taken from the parameterization of Gao et al. ${ }^{26}$, which is based on observations from Bertaux et al. ${ }^{25}$ and the Venera 11,13 and 14 missions ${ }^{24}$.

Atmospheric relative humidity is a ratio, calculated according to the partial pressure of the atmospheric water vapour divided by the saturation vapour pressure of liquid water at the corresponding temperature, which we computed with the parameterization of Murphy and Koop ${ }^{50}$. The results (Table 1) are shown pictorially in Fig. 2 where the uncertainty in altitude is smaller than the size of the data-point markers. The uncertainty in relative humidity was determined by combining the uncertainties in temperature, pressure and water-vapour mixing ratio. It was dominated by the uncertainty in the water-vapour parameter which, based on the comparison of the fit to observations shown in Gao et al. ${ }^{26}$, we estimate to be $\pm 30 \%$ of the plotted values (Fig. 2). It is relevant to note that the data directly determine the water-vapour mixing ratio to be $1 \mathrm{ppm}$ at $70-90 \mathrm{~km}$ and 30 ppm below $60 \mathrm{~km}$. In the Gao et al. parameterization ${ }^{26}$, the transition is smooth, and the water-vapour mixing ratio decreases sharply above $60 \mathrm{~km}$ resulting in a peak in the relative humidity at about $65 \mathrm{~km}$. If instead the water-vapour mixing ratio is held at $30 \mathrm{ppm}$ through the cloud layer until $70 \mathrm{~km}$, the atmospheric relative humidity would rise monotonically to $0.07 \%$ at $70 \mathrm{~km}$.

The water activity of the droplets (Table 1 ) is taken as equivalent to the relative humidity of the atmosphere. This is because the time constant for growth of droplets (due to collisions with the ambient water vapour) is short compared with droplet transport times. This assumption is common in Venus atmospheric models $\mathrm{s}^{29}$. The droplets are large enough (radius approximately $1 \mu \mathrm{m}$ ) that the reduction of water activity due to curvature in the droplet-the Kelvin effect-is negligible $e^{62,63}$. The timescale for water-vapour molecules to accrete on a cloud droplet can be estimated from the collision rate as derived from kinetic theory.

In the kinetic approximation, the number of atoms sticking on a droplet of radius $r$ per unit time is approximately $\alpha n 4 \pi r^{2}\left(k_{\mathrm{B}} T / 2 \pi m\right)^{1 / 2}\left(\right.$ ref. $\left.{ }^{64}\right)$, where $\alpha$ is the mass accommodation coefficient, $n$ is the density of gas-phase water molecules in the atmosphere, $k_{\mathrm{B}}$ is Boltzmann's constant, $T$ is the temperature and $m$ is the mass of the water molecules. The formula above ignores the correction due to the small mean free path. Over the range of altitudes considered here, the mean free path varies from 0.02 to $1.2 \mu \mathrm{m}$ which is smaller than, or comparable to, the droplet size of about $1 \mu \mathrm{m}$ radius.

Expressed in terms of the $e$-folding time, for the droplet to grow in size by a factor of $e$, we have $\tau^{-1}=r^{-1} \mathrm{~d} r / \mathrm{d} t=r^{-1} \alpha(n / \rho)\left(m k_{\mathrm{B}} T / 2 \pi\right)^{1 / 2}$, where $\rho$ is the density of the droplet and $\mathrm{d} r / \mathrm{d} t$ is the rate of change of the radius with time, $t$. If we evaluate this for representative conditions in the atmospheric profile, pressure $\sim 1 \mathrm{~atm}$, temperature $\sim 0{ }^{\circ} \mathrm{C}$ and water-vapour concentration $\sim 20 \mathrm{ppm}$, we obtain a value of $\tau=0.4 \mathrm{~s}$, for $\alpha=1$. The mass accommodation, $\alpha$, is typically assumed to be unity in models of Venus cloud physics ${ }^{26}$. However, Gardner et al ${ }^{65}$ reported a lower limit to $\alpha$ of $(5.4 \pm 0.6) \times 10^{-2}$ based on laboratory experiments, which gives a value of $\tau=0.8 \mathrm{~s}$. These values of $\tau$ can be compared to the time for a $1-\mu \mathrm{m}$-radius droplet to settle $1 \mathrm{~km}$, which is the step size in our atmospheric model. The settling velocity of a 1- $\mu \mathrm{m}$-radius droplet at standard pressure is $\sim 10^{-3} \mathrm{~cm} \mathrm{~s}^{-1}$ giving a time of $10^{8} \mathrm{~s}$ to cover $1 \mathrm{~km}$. Eddy mixing will be more important for droplet transportation than rates of settling. Zhang et al. ${ }^{29}$ give an eddy coefficient, $D$, at $60 \mathrm{~km}$ of $4 \times 10^{4} \mathrm{~cm}^{2} \mathrm{~s}^{-1}$. Transport across a distance $Z$ will occur over a timescale of $\sim Z^{2} / D$. For $Z=1 \mathrm{~km}$, giving a transport time of $2.5 \times 10^{5} \mathrm{~s}$. This calculation supports the conclusion that the cloud droplets are in balance with the local atmospheric water abundance, and we equate the water activity in the droplets with the atmospheric 
relative humidity in Table 1 . The low values of water activity that we find (Table 1 ) are consistent with atmospheric models (Fig. A4 in ref. ${ }^{29}$ ).

$\mathrm{H}_{2} \mathrm{SO}_{4}$ concentration in the droplets of Venus's clouds. The functional capability of cellular systems is determined by the biophysical and physicochemical conditions to which the cell-not least, the plasma membrane-are exposed assuming that biocidal factors are absent, and nutrients and energy sources are available. Therefore, it is imperative to consider habitability at a scale that is pertinent to the microbial cell and its macromolecular systems rather than confining analyses to a macro level or planetary scale, or an anthropocentric viewpoint. Factors such as availability of nutrients, energy sources, temperature, cosmic rays and ultraviolet radiation have been considered elsewhere (for example, refs. ${ }^{5,11,13,66,67}$ ), so here we focused on quantifying the sulfuric acid concentration and water activity of the droplets in Venusian clouds.

The sulfuric acid concentration within cloud droplets was calculated assuming that the primary sulfate species is sulfuric acid. We used the Gmitro and Vermeulen ${ }^{20,21}$ data to calculate the sulfuric acid concentrations that are in equilibrium with the relative humidity in Venus's atmosphere, indicated by grey circles in Fig. 1; see also Table 1. We also used the E-AIM ${ }^{19}$, although the relative humidity values are below 0.01 water activity ( $1 \%$ relative humidity), which is the lower water-activity limit at which the model runs. The values that we obtained agreed with those calculated using the Gmitro and Vermeulen ${ }^{20,21}$ data, to within $\pm 1 \%\left(\mathrm{w} / \mathrm{w}\right.$ ) sulfuric acid for temperatures between $-40^{\circ} \mathrm{C}$ and $25^{\circ} \mathrm{C}$. The formation of solids is believed not to occur, so was suppressed in the model. If we assumed that there is an uncertainty of $\pm 30 \%$ in relative humidity, this leads to only minor changes in sulfuric acid concentrations, which were always less than the symbol size of the grey circles in Fig. 1.

Acidity and water-activity limits for active life on Earth. No individual terrestrial microbe is likely capable of metabolism under the combined conditions of 0.585 water activity, extreme acidity, high ultraviolet radiation, and limited nutrients and energy sources. Some archaea can grow down to $\mathrm{pH} 1$ in the range $70^{\circ} \mathrm{C}$ to $80^{\circ} \mathrm{C}$, which is equivalent to a sulfuric acid concentration of about $1.2 \%(\mathrm{w} / \mathrm{w})$ according to Clegg et al..$^{19}$. Above $50^{\circ} \mathrm{C}$, there are no known microbes able to grow (or retain metabolic functions) at or below a water activity of about 0.700 (the water activity of saturated $\mathrm{NaCl}$ at $50{ }^{\circ} \mathrm{C}$ is 0.745 (ref. ${ }^{68}$ )); see Fig. $1 \mathrm{~A}$ of Lee et al. ${ }^{69}$ and Fig. 1 of Harrison et al..$^{70}$. Nevertheless, 0.585 does represent the ultimate water-activity limit based on current knowledge (see above); we therefore show the position of this water-activity value in Fig. 1. For further details, see Supplementary text on biophysical limits for terrestrial microbes.

The fungus that is able to differentiate and grow at 0.585 , the lowest water activity for active life $\mathrm{e}^{14,32}$ (A. penicillioides), is also acidotolerant; see below. At this water activity, differentiation and cell division were observed on a nutrient medium supplemented with 7.7 M glycerol, at pH6.1 (ref. ${ }^{14}$ ). Low water activity, low-pH habitats in nature are not common. Although a recent study of acid-brine lakes (Western Australia) revealed water-activity values as low as 0.714 ( $\mathrm{pH} 1.4$ ), there are no definitive data to indicate microbial activity under these conditions ${ }^{71}$. The lowest water activity at which proliferation of halophilic prokaryotes has been observed is 0.635 , in a NaCl-dominated but $\mathrm{MgCl}_{2}$-rich bittern brine ${ }^{3}$. We doubt that any acidophilic species would be capable of metabolism at such biologically hostile water-activity values given the level of energy generation that would be needed to cope with concomitant extremes of water activity and acidity. Also, the cellular damage caused by this combination of extremes are likely to outweigh the capacity for self-repair, resulting in senescence and death ${ }^{72}$. It is possibly for the same reason that the most-extreme xerophiles and halophiles have not evolved to be acidophilic, even though in nature they can be exposed to low-pH conditions (for example, in acid-brine lakes and bittern brines).

The acid tolerance limit of the most acidophilic microbe known (P. torridus), detailed above, has been converted to a water-activity value as shown in Fig. 3. There is one acidophile that is known to be halotolerant; the bacterium A. aeolianus. This microbe is chemolithoautotrophic and capable of growth at $1,283 \mathrm{mM} \mathrm{NaCl}$ and $\mathrm{pH} 2$ (adjusted by addition of concentrated sulfuric acid to the nutrient medium; $30^{\circ} \mathrm{C}$; refs. ${ }^{73,74}$ ). In these studies, the final concentration of sulfuric acid was not empirically determined. Therefore, we used the E-AIM ${ }^{19}$ to calculate the concentration of sulfuric acid that corresponds to a $\mathrm{pH}$ of 2 at $30^{\circ} \mathrm{C}$; this was $0.071 \%(\mathrm{w} / \mathrm{w})$. Also using the E-AIM, we calculated that this concentration of sulfuric acid causes a water-activity reduction of 0.0003 units. We then calculated the water activity of the nutrient medium, which is a sum of the water-activity changes caused by sulfuric acid, $\mathrm{NaCl}$, nutrients and agar. The water-activity reduction caused by $1,283 \mathrm{mM} \mathrm{NaCl}$ is 0.043 units $^{75}$, and the reduction caused by nutrients and agar in the culture medium is typically 0.002 units. Therefore, the water activity of the medium $(1-[0.0003+0.043+0.002])$ was 0.955 , as shown in Fig. 3. Whereas the related bacterium Acidihalobacter prosperus grew down to $\mathrm{pH} 1.5$ in a separate experiment, it is not known to be capable of this level of acid tolerance at elevated $\mathrm{NaCl}^{73}$.

The 0.585 water-activity limit for A. penicillioides growing at a high glycerol concentration (at $24^{\circ} \mathrm{C}(297 \mathrm{~K})$, see black triangle in Fig. 1) would be equivalent to about $39 \%(\mathrm{w} / \mathrm{w})$ sulfuric acid according to Gmitro and Vermeulen ${ }^{20,21}$ and the $\mathrm{E}^{-\mathrm{AIM}^{19}}{ }^{19}$, and a molality-based $\mathrm{pH}$ value of -1.55 according to the E-AIM and the
IUPAC convention ${ }^{61}$. However, growth of $A$. penicillioides has only been recorded down to $\mathrm{pH} 3$ (on a citric acid/ $\mathrm{Na}_{2} \mathrm{PO}_{4}$-buffered nutrient medium) according to a study of germination over a range of $\mathrm{pH}$ (Supplementary Fig. 2 and ref. ${ }^{76}$ ). Therefore, we carried out an extrapolation of the data shown in Supplementary Fig. 2 to determine the theoretical $\mathrm{pH}$ minimum for growth, using the Cardinal $\mathrm{pH}$ Model (equation (7) from Rosso et al. ${ }^{77}$ ) in R version 4.0.3 (Supplementary Fig. 2). From the datapoints in Supplementary Fig. 2, the $\mathrm{pH}$ beyond which no germ-tube growth occurs $\left(\mathrm{pH}_{\max }\right)$ was set at 10 ; the $\mathrm{pH}$ below which no germ-tube growth occurs $\left(\mathrm{pH}_{\min }\right)$ was provisionally set at 2 ; the $\mathrm{pH}$ at which that rate of germ-tube growth was optimal $\left(\mathrm{pH}_{\text {opt }}\right)$ was set at 6.5 ; and the optimum rate of germ-tube extension $\left(\mu_{\text {opt }}\right)$ was set at $0.0188 \mathrm{~mm} \mathrm{~d}^{-1}$ to obtain a curve (Rosso et al. $\left.{ }^{77}\right)$. The fit of this curve was refined using the sum of square residuals (SSR) formula and then a nonlinear minimization formula, resulting in an SSR value of $1.07 \times 10^{-6}$. Using this procedure, the minimum $\mathrm{pH}$ was found to be 2.3 (Supplementary Fig. 2). To assess the confidence of this value, a $95 \%$ confidence level was determined using this formula ${ }^{78}$

$$
\frac{\theta}{S(\theta)} \leq S(\hat{\theta})\left(1+\frac{p}{n-p} F_{p ; n-p}^{\alpha}\right)
$$

where $S$ is the sum of squared residuals; $F$ denotes the Fisher distribution; $\alpha$ indicates a region of confidence for the value of the parameters with a risk of the first kind that is given by the set of values of parameters such that the sum of the SSR does not exceed a given threshold; $p$ is the number of model parameters; $n$ is the number of points available in the dataset; and $\hat{\theta}$ is the vector of values of parameters such that the criterion is minimal. The $95 \%$ confidence interval for the minimum $\mathrm{pH}$ for growth was $\mathrm{pH} 1.96$ to 2.57 .

The $\mathrm{pH}$ value 2.3 is equivalent to $0.031 \%$ (w/w) sulfuric acid at $24^{\circ} \mathrm{C}$ according to Clegg et al. ${ }^{19}$. Furthermore, the sulfuric acid concentration of $39 \%$ (w/w) ( $\equiv 0.585$ water activity) is about half that of the least concentrated of Venus's cloud droplets (see below) and about four times the tolerance limit of the most acidophilic microorganism known.

The sulfuric acid concentrations shown on the vertical axis of Fig. 3 were first calculated using the E-AIM ${ }^{19}$ and Gmitro and Vermeulen ${ }^{20,21}$ at $25^{\circ} \mathrm{C}$, and the average concentration was then plotted. Whereas there is some temperature dependence of the relationship between sulfuric acid concentration and water activity, this is of minor importance on the scale of Fig. 3 (see Supplementary Fig. 1 and Supplementary Tables 1 and 2 for temperature comparisons). The variation in water activity over the entire temperature range of Venus's cloud layer is from 0.0037 (at $-25^{\circ} \mathrm{C}$ ) to 0.00003 (at $130^{\circ} \mathrm{C}$ ). On the linear water-activity scale in Fig. 3 ; this variation occurs within the red zone (that appears as a thick red line) and so is in this way indistinguishable.

\section{Activities of sulfuric acid on the cellular system. We sought to identify the} primary activities of sulfuric acid on the cellular system because these may also act as determinants for habitability of sulfuric acid clouds. Broadly, they fell into three logical categories: those relating to the inherent properties of the $\mathrm{H}_{2} \mathrm{SO}_{4}$ $\mathrm{H}_{2} \mathrm{O}$ mixtures, the biophysical effects on cellular macromolecules (operating at the level of non-covalent interactions) and chemical modification(s) of cellular macromolecules (these operate primarily at the level of covalent bonds). Searches of the literature (physics, chemistry, biochemistry, microbiology) revealed the primary modes of action of sulfuric acid (see Supplementary text for Fig. 3). These were used to construct a schematic of habitability for the sulfuric acid clouds of Venus's putative habitable zone (Fig. 3). This display shows: properties of $\mathrm{H}_{2} \mathrm{SO}_{4}-\mathrm{H}_{2} \mathrm{O}$ mixtures; biophysical effects of sulfuric acid on cellular macromolecules; chemical modifications of cellular macromolecules induced by sulfuric acid; and the known tolerance limits of terrestrial life in relation to acidity (acidophile and polyextremophile) and low water activity (xerophile/polyextremophile and halophiles). It also shows the sulfuric acid concentration of the putative habitable zone of the Venusian clouds (red zone, Fig. 3).

Determination of habitability for Venus's acid clouds. The notion that phosphine in the Venusian atmosphere might act as a biosignature rests on the fact that no long-lived geological processes on Earth are known to generate it, whereas it may be produced by terrestrial organisms ${ }^{79}$. The question is, however, whether Venus hosts any regimes that might plausibly support life. In the current study, determinations of habitability of Venus's clouds were based on knowledge of:

- activities of sulfuric acid on the cellular system;

- the current understanding that cellular life requires water ${ }^{16,34,35}$,

- biophysical/physicochemical limits for life on Earth and the underlying stress mechanisms that ultimately induce cell-system failure;

- the sulfuric acid concentration and water activity of droplets within Venus's clouds at the altitude/temperature range that is potentially habitable, based on knowledge of the functional biosphere on Earth; and

- identification of any thermodynamic/physicochemical distance (along with the acidity and water-activity scales) between the metabolic activity limits of terrestrial life and the putative habitat of Venus's clouds. These are summarized in Table 1 and Fig. 3. 
One must assume that, at the acid concentrations found in Venus's clouds, sulfuric acid molecules would hydrogen-bond to biological macromolecules (Fig. 3) if these are at all comparable to hydrophilic proteins in as much as they are water soluble; certainly, hydrogen bonding of sulfuric acid to water and methanol is possible ${ }^{80,81}$. In this case, solvation would more closely resemble that in the presence of concentrated macromolecular cryoprotectants such as trehalose, where the role of the protectant co-solvent is more to immobilize the protein and prevent denaturation than to sustain function.

At the acidity limit for terrestrial life, we believe that, paradoxically, it is water activity that is the limiting factor. The water activity of a $11.5 \%(\mathrm{w} / \mathrm{w})$ sulfuric acid solution according to the Clegg et al. ${ }^{19}$ thermodynamic model is about 0.95 (we calculated $11.4 \%(\mathrm{w} / \mathrm{w})$ at $56^{\circ} \mathrm{C}(329 \mathrm{~K})$; that is, near the upper limit of the model, and extrapolation of values derived from Clegg et al. to $60^{\circ} \mathrm{C}$ yields $11.5 \%(\mathrm{w} / \mathrm{w})$ ). The majority of microbes, apart from specialist halophiles and xerophiles, have a minimum water activity for growth in the range 0.920 to 0.950 (refs. ${ }^{4,16,82}$ ), and a likely limit in this range is consistent with the growth phenotype and ecology of Picrophilus, which is not known in saline habitats. The sulfuric acid tolerance of Picrophilus, therefore, is probably determined by its limits of xerotolerance rather than tolerance to acidity per se. This is analogous to the growth limit of the Saccharomyces cerevisiae strains that are able to tolerate the highest levels of ethanol, a cellular stressor that entropically disorders macromolecular systems; that is, it acts as a chaotrope ${ }^{83}$. The apparent tolerance limit to this chaotropicity also coincides with the lower water-activity limit for growth of this species (see below). It is noteworthy that the highest sulfuric acid tolerance reported for a microbe was exhibited at a high temperature $\left(60^{\circ} \mathrm{C}\right)$, which is in the thermophile range; and that some acidophiles even grow up to 80 or $90^{\circ} \mathrm{C}$. Whereas chaotropic substances can reduce the growth minima of microbes ${ }^{36}$, diverse lines of evidence suggest that kosmotropic substances (for example, sulfate ions) can stabilize cellular macromolecules at high temperature and may thereby enable growth at higher temperatures; conversely, increased temperatures probably enable tolerance to high concentrations of kosmotropes (ref. ${ }^{84}$ and references therein; ref. ${ }^{85}$ ). For the ultimate terrestrial acidophile, water activity and $\mathrm{pH}$ may act concomitantly to curtail metabolism.

Other biophysical conditions that appear to limit the functional biosphere on Earth might also be a consequence of insufficient solvent water. For example, the growth limit of A. penicillioides at $7.7 \mathrm{M}$ glycerol may be an artefact in as much as the microbe may be capable of growth at water activities below the 0.585 value. In this case, when glycerol greatly predominates over water, the cell may cease to function. It is noteworthy that other studies of xerophilic fungi in a similar range of glycerol concentrations found that substituting some of the glycerol with a different solute could reduce the water-activity limit for growth ${ }^{86,87}$. Whereas this could have been because the kosmotropicity of the added solute mitigated against the chaotropicity of glycerol $^{87}$, it is plausible that the partial substitution of glycerol by a different solute meant that a critical concentration (where this polyol prevents water acting as the cellular solvent) was not reached.

Similarly, the most ethanol-tolerant strains of $S$. cerevisiae remain active up to almost $20 \%(\mathrm{w} / \mathrm{v})($ that is, $28 \%(\mathrm{v} / \mathrm{v}))$ ethanol; concentrations that reduce water activity to a level consistent with their limit of xerotolerance; about 0.900 water activity $^{84}$. Studies of ethanol-water mixtures show qualitative changes in this range, which have been discussed in the context of microheterogeneity ${ }^{88}$, but can equally be considered an indicator that ethanol has begun to displace water as the cellular solvent. The same may be true for the ions that can limit the growth of even the most-extreme halophiles; this may also be why the water-activity limits of extremely halophilic prokaryotes (in brines) and extremely xerophilic eukaryotes (in non-saline habitats) converge towards a common value ${ }^{3}$. The issue in each case (brines, sulfuric acid, glycerol, ethanol) seems to be not so much the amount of co-solvent that the microbial cell can tolerate, but how little water remains.

The situation would be further complicated for sulfuric acid by the likely extreme protonation of any protein-like macromolecules, and perhaps by the disruptive 'salting out' effect exerted on proteins by sulfate- and hydrogen sulfate ions, among other adverse effects (Fig. 3). Might sulfuric acid itself act as a biophilic solvent, though? There seems no reason to rule out this speculative possibility per se. Sulfuric acid is capable of forming hydrogen bonds with water molecules, hydroxyl groups and carboxylic acids ${ }^{80,89}$, and it is a polar molecule with a dielectric constant comparable to that of water (around 100 and 80, respectively). But it cannot be expected to mediate macromolecular interactions in the same way that water does. The role of water's motions and fluctuations on biological macromolecules seems to depend, for example, on the highly cooperative dynamics created by its three-dimensional hydrogen-bonding network. So, there would then be little justification for extrapolating from the biochemistry of terrestrial organisms at all.

Analyses of water activity and habitability for clouds of Jupiter, Mars and exoplanets. For Jupiter, the method employed consisted of three steps. First, a measured profile of temperature and pressure (from the Galileo Atmospheric Entry Probe) was used; second, we assumed that the water abundance is set at the value that corresponds to the solar ratio of oxygen to hydrogen; and third, water activity was calculated by computing the atmospheric partial pressure of water from the pressure and water-vapour mixing ratio, and the equilibrium vapour pressure of liquid water from the temperature. Water activity is the ratio of these. Below freezing, when the calculation indicates that ice is present, the water activity is equal to the ratio of the vapour pressure of ice to the vapour pressure of pure liquid water. This ratio is also how the water activity at Martian temperatures was computed. The method employed for Jupiter can be applied to exoplanets when the temperature and pressure profiles and water abundance are obtained from observations of a planet's transits in front of the star.

Analyses of water activity and habitability of Earth's clouds. As a comparitve case study, we applied our approach of computing the water activity from temperature and water-vapour abundance data also to Earth's atmosphere and clouds. For this purpose, we used the Committee on Space Research (COSPAR) International Reference Atmosphere ${ }^{90}$ that acts as a model of the terrestrial atmosphere and is based on empirical temperature, pressure and altitude data. Both temperature and water-vapour mixing ratio are highly variably in Earth's atmosphere. This variability is to a large part due to the existence of both land and expansive oceans, differences in solar radiative influx with latitude, and the pronounced diurnal light-dark cycle and annual seasonal cycle. Indeed, thunderstorms and dry heatwaves can occur at the same location within a matter of days. Therefore, we used three representative water-vapour mixing ratios-for the polar, mid-latitude and tropical troposphere-(solid blue, solid green, and solid red circles respectively, in Fig. 5) ${ }^{91}$ and one average profile for altitudes of $\geq 20 \mathrm{~km}$ (solid black circles in Fig. 5), which is based on data from Bohren and Clothiaux ${ }^{92}$. As the height of the interface between the troposphere and stratosphere (tropopause) varies with latitude, being lower at the poles and higher in the tropics, we interpolated the tropospheric profiles to the water-vapour mixing ratio at $20 \mathrm{~km}$ (open circles in Fig. 5)

These water-vapour mixing ratios can then be used together with the COSPAR International Reference Atmosphere ${ }^{90}$ average temperature and pressure profiles to calculate relative humidity and water activity. For this purpose, we extracted temperature-versus-altitude profiles corresponding to each January and July for the polar region at $80^{\circ} \mathrm{N}$ and $80^{\circ} \mathrm{S}$, mid-latitude region at $40^{\circ} \mathrm{N}$ and $40^{\circ} \mathrm{S}$ and tropical region at $10^{\circ} \mathrm{N}$ and $10^{\circ} \mathrm{S}$ (that is, 12 profiles in total). We did the same for the corresponding atmospheric pressure-versus-altitude profiles. As the COSPAR pressure data only reach from 20 to $120 \mathrm{~km}$, the pressure data were smoothly extrapolated from 20 to $0 \mathrm{~km}$ using the slope of the 1976 US Standard Atmosphere at a resolution of $5 \mathrm{~km}$ (obtained from Table 1 in part 4 of ref. ${ }^{93}$ ). From these data, we calculated the water partial pressure for each of the above profiles, and the vapour pressure of liquid water ${ }^{50}$ using the temperature at each altitude. The definitions of the ratio of water partial pressure to liquid-water-vapour pressure and relative humidity are equivalent. Calculations of the former, therefore, yield the corresponding relative humidity profiles. In Fig. $6 \mathrm{a}$, we show the range of relative humidity for each of the three regions (polar, mid-latitude, tropical), in each case using the maximum and minimum relative humidity values of each of the four temperature-versus-altitude profiles of each region as the bounding values.

In Earth's troposphere, relative humidity is highly variable, both temporally and spatially. For our analysis of monthly average profiles, the largest variation occurs in the polar regions (Fig. 6a). At higher altitudes, in the stratosphere and mesosphere, relative humidity is very low except for a local maximum in the summer polar mesosphere at about $85 \mathrm{~km}$ altitude, and a local maximum in the winter polar stratosphere at an altitude of about $25 \mathrm{~km}$. Given that the stratosphere and mesosphere are very dry, clouds occur only during exceptionally cold periods in the polar regions. Typical conditions for cloud formation in Earth's atmosphere are detailed in Supplementary Table 3.

As in our analysis of Venus's clouds (above), the ambient relative humidity dictates the water activity of airborne microorganisms and liquid aerosol particles that may harbour life forms: under equilibrium conditions, which are often readily met in Earth's atmosphere, relative humidity and water activity are equivalent. Therefore, overall, the relative humidity profiles in Fig. 6 indicate that the water-activity values are on average too low for active life (that is, $<0.585$ ) in the middle and upper stratosphere and the mesosphere. The maximum water activity at altitudes of $30 \mathrm{~km}$ and $80 \mathrm{~km}$ was about 0.003 and 0.006 , respectively, and it was below $10^{-4}$ to $10^{-5}$ at altitudes in between. In contrast, high water-activity values occur frequently in the troposphere at nearly all altitudes, even for the latitudinal monthly averages used here. We note, however, that other factors (including ultraviolet radiation) impact microbial vitality and survival, and that these relative humidity profiles are more representative of average cloud-free conditions, and the actual variability is certainly much larger than the range indicated in Fig. 6a. This is seen best by the average mid-latitudinal profiles (green area in Fig. 6a), which are nearly always below a water activity of about 0.420 ( $42 \%$ relative humidity). Similarly, water-activity values are below about 0.60 (60\% relative humidity) for the average tropical profiles at altitudes of about $10 \mathrm{~km}$ and below (red in Fig. 6a). Such low values would imply that liquid-water clouds, which require water-activity values of about 1 ( $100 \%$ relative humidity), would not be able to form in the mid-latitudes or the lower and middle tropical troposphere. Clearly, this predication is not faithful to reality and indicates a dry bias when using monthly and latitudinal average profiles. In fact, in the tropical and mid-latitude regions of the lower and middle troposphere, we would expect values of water activity of up to $1(100 \%$ relative humidity). For these reasons, we used an alternative approach 
to estimate the typical water-activity values that occur within clouds (Fig. 6b and 'Supplementary text for Fig. 6b).

\section{Data availability}

We confirm that all relevant data are included in the paper and/or its Supplementary Information files. Source data are provided with this paper.

Received: 15 October 2020; Accepted: 7 May 2021; Published online: 28 June 2021

\section{References}

1. DeLeon-Rodriguez, N. et al. Microbiome of the upper troposphere. Proc. Natl Acad. Sci. USA 110, 2575-2580 (2013).

2. Kabir, E. et al. Recent advances in monitoring, sampling, and sensing techniques for bioaerosols in the atmosphere. ACS Sens. 5, 1254-1267 (2020).

3. Stevenson, A. et al. Is there a common water-activity limit for the three domains of life? ISME J. 9, 1333-1351 (2015)

4. Rummel, J. D. et al. A new analysis of Mars "special regions": findings of the Second MEPAG Special Regions Science Analysis Group (SR-SAG2). Astrobiology 14, 887-968 (2014).

5. Greaves, J. S. et al. Phosphine gas in the cloud decks of Venus. Nat. Astron. https://doi.org/10.1038/s41550-020-1174-4 (2020)

6. Mogul, R., Limaye, S. S., Way, M. J. \& Cordova, J. A. Venus' mass spectra show signs of disequilibria in the middle clouds. Geophys. Res. Lett. 48, e2020GL091327 (2021).

7. Morowitz, H. \& Sagan, C. Life in the clouds of Venus? Nature 215, 1259-1260 (1967)

8. Limaye, S. S. et al. Venus' spectral signatures and the potential for life in the clouds. Astrobiology 18, 1181-1198 (2018).

9. Izenberg, N. R. et al. The Venus life equation. Astrobiology https://doi. org/10.1089/ast.2020.2326 (2021).

10. Sagan, C. The planet Venus. Science 133, 849-858 (1961)

11. Cockell, C. S. Life on Venus. Planet. Space Sci. 47, 1487-1501 (1999).

12. Bains, W. et al. Phosphine on Venus cannot be explained by conventional processes. Preprint at http://arxiv.org/abs/2009.06499 (2020).

13. Seager, S. et al. The Venusian lower atmosphere haze as a depot for desiccated microbial life: a proposed life cycle for persistence of the Venusian aerial biosphere. Astrobiology https://doi.org/10.1089/ast.2020.2244 (2020).

14. Stevenson, A. et al. Aspergillus penicillioides differentiation and cell division at 0.585 water activity. Environ. Microbiol. 19, 687-697 (2017).

15. Meisner, A. et al. Soil microbial legacies differ following drying-rewetting and freezing-thawing cycles. ISME J. 15, 1207-1221 (2021)

16. Stevenson, A. et al. Multiplication of microbes below 0.690 water activity: implications for terrestrial and extraterrestrial life. Envrion. Microbiol. 17, 257-277 (2015).

17. Benner, S. A., Ricardo, A. \& Carrigan, M. A. Is there a common chemical model for life in the Universe? Curr. Opin. Chem. Biol. 8, 672-689 (2004)

18. Schleper, C. et al. Picrophilus gen. nov., fam. nov.: a novel aerobic, heterotrophic, thermoacidophilic genus and family comprising archaea capable of growth around pH 0. J. Bacteriol. Res. 177, 7050-7059 (1995).

19. Clegg, S. L., Brimblecombe, P. \& Wexler, A. S. Thermodynamic model of the system $\mathrm{H}^{+}-\mathrm{NH}_{4}{ }^{+}-\mathrm{SO}_{4}{ }^{2-}-\mathrm{NO}_{3}{ }^{-}-\mathrm{H}_{2} \mathrm{O}$ at tropospheric temperatures. J. Phys. Chem. A 102, 2137-2154 (1998).

20. Gmitro, J. I. \& Vermeulen, T. Vapor-liquid equilibria for aqueous sulfuric acid. AIChE J. 10, 740-746 (1964).

21. Gmitro, J. I. \& Vermeulen, T. Vapor-Liquid Equilibria for Aqueous Sulfuric Acid Technical Report No. UCRL-10886; TID-4500 (Ernest Orlando, Lawrence Berkeley National Laboratory, 1963); https://doi.org/10.2172/876220

22. Wilson, R. E. Humidity control by means of sulfuric acid solutions, with critical compilation of vapor pressure data. J. Indust. Eng. Chem. 13, 326-331 (1921).

23. Seiff, A. et al. Models of the structure of the atmosphere of Venus from the surface to 100 kilometers altitude. Adv. Space Res. 5, 3-58 (1985).

24. Ignatiev, N. I. et al. Water vapour in the lower atmosphere of Venus: a new analysis of optical spectra measured by entry probes. Planet. Space Sci. 45, 427-438 (1997).

25. Bertaux, J. L. et al. SPICAV on Venus Express: three spectrometers to study the global structure and composition of the Venus atmosphere. Planet. Space Sci. 55, 1673-1700 (2007).

26. Gao, P., Zhang, X., Crisp, D., Bardeen, C. G. \& Yung, Y. L. Bimodal distribution of sulfuric acid aerosols in the upper haze of Venus. Icarus 231, 83-98 (2014)

27. Carslaw, K. S., Peter, T. \& Clegg, S. L. Modeling the composition of liquid stratospheric aerosols. Rev. Geophys. 35, 125-154 (1997).

28. Martin, S. T. Phase transitions of aqueous atmospheric particles. Chem. Rev. 100, 3403-3454 (2000)

29. Zhang, X., Liang, M. C., Mills, F. P., Belyaev, D. A. \& Yung, Y. L. Sulfur chemistry in the middle atmosphere of Venus. Icarus 217, 714-739 (2012).
30. Arney, G. et al. Spatially resolved measurements of $\mathrm{H}_{2} \mathrm{O}, \mathrm{HCl}, \mathrm{CO}, \mathrm{OCS}, \mathrm{SO}_{2}$ cloud opacity, and acid concentration in the Venus near-infrared spectral windows. J. Geophys. Res. Planets 119, 1860-1891 (2014).

31. Krasnopolsky, V. A. Vertical profiles of $\mathrm{H}_{2} \mathrm{O}, \mathrm{H}_{2} \mathrm{SO}_{4}$, and sulfuric acid concentration at 45-75 km on Venus. Icarus 252, 327-333 (2015).

32. Hallsworth, J. E. Wooden owl that redefines Earth's biosphere may yet catapult a fungus into space. Environ. Microbiol. 21, 2202-2211 (2019).

33. Koop, T., Luo, B., Biermann, U. M., Crutzen, P. J. \& Peter, T. Freezing of $\mathrm{HNO}_{3} / \mathrm{H}_{2} \mathrm{SO}_{4} / \mathrm{H}_{2} \mathrm{O}$ solutions at stratospheric temperatures: nucleation statistics and experiments. J. Phys. Chem. A 101, 1117-1133 (1997).

34. Ball, P. Water is an active matrix of life for cell and molecular biology. Proc. Natl Acad. Sci. USA 114, 13327-13335 (2017).

35. Brown, A. D. Microbial Water Stress Physiology (John Wiley, 1990).

36. Chin, J. P. et al. Solutes determine the temperature windows for microbial survival and growth. Proc. Natl Acad. Sci. USA 107, 7835-7840 (2010).

37. Hallsworth, J. E., Heim, S. \& Timmis, K. N. Chaotropic solutes cause water stress in Pseudomonas putida. Environ. Microbiol. 5, 1270-1280 (2003).

38. de Lima Alves, F. et al. Concomitant osmotic and chaotropicity-induced stresses in Aspergillus wentii: compatible solutes determine the biotic window. Curr. Genet. 61, 457-477 (2015).

39. Santos, R., de Carvalho, C. C. C. R., Stevenson, A., Grant, I. R. \& Hallsworth J. E. Extraordinary stress-tolerance of mycobacteria. Environ. Microbiol. Rep. 7, 746-764 (2015).

40. Oleinikova, A., Smolin, N., Brovchenko, I., Geiger, A. \& Winter, R. Formation of spanning water networks on protein surfaces via 2D percolation transition. J. Phys. Chem. B 109, 1988-1998 (2005).

41. Arcangeli, C., Bizzarri, A. R. \& Cannistraro, S. Role of interfacial water in the molecular dynamics-stimulated dynamical transition of plastocyanin. Chem. Phys. Lett. 291, 7-14 (1998).

42. Roos-Serote, M. et al. Proximate humid and dry regions in Jupiter's atmosphere indicate complex local meteorology. Nature 405, 158-160 (2000)

43. Seiff, A. et al. Thermal structure of Jupiter's atmosphere near the edge of a $5-\mu \mathrm{m}$ hot spot in the north equatorial belt. J. Geophys. Res. Planets 103, 22857-22889 (1998).

44. Wong, M. H., Mahaffy, P. R., Atreya, S. K., Niemann, H. B. \& Owen, T. C. Updated Galileo probe mass spectrometer measurements of carbon, oxygen, nitrogen, and sulfur on Jupiter. Icarus 171, 153-170 (2004).

45. Li, C. et al. The water abundance in Jupiter's equatorial zone. Nat. Astron. 4, 609-616 (2020).

46. Roos-Serote, M., Atreya, S. K., Wong, M. K. \& Drossart, P. On the water abundance in the atmosphere of Jupiter. Planet. Space Sci. 52, 397-414 (2004)

47. Schuerger, A. C., Richards, J. T., Newcombe, D. A. \& Venkateswaran, K. Rapid inactivation of seven Bacillus spp. under simulated Mars UV irradiation. Icarus 181, 52-62 (2006).

48. Withers, P. \& Smith, M. D. Atmospheric entry profiles from the Mars exploration rovers Spirit and Opportunity. Icarus 185, 133-142 (2006).

49. Fedorova, A. A. et al. Stormy water on Mars: The distribution and saturation of atmospheric water during the dusty season. Science 367, 297-300 (2020).

50. Murphy, D. M. \& Koop, T. Review of the vapour pressures of ice and supercooled water for atmospheric applications. Q. J. R. Meteorol. Soc. 131, 1539-1565 (2005).

51. Kreidberg, L. et al. A precise water abundance measurement for the hot Jupiter WASP-43b. Astrophys. J. Lett. 793, L27 (2014).

52. Villanueva, G. et al. No phosphine in the atmosphere of Venus. Preprint at https://arxiv.org/abs/2010.14305 (2020)

53. Snellen, I. A. G., Guzman-Ramirez, L., Hogerheijde, M. R., Hygate, A. P. S. \& van der Tak, F. F. S. Re-analysis of the $267 \mathrm{GHz}$ ALMA observations of Venus-no statistically significant detection of phosphine. Astron. Astrophys. 644, L2 (2020).

54. Witze, A. 'Life on Venus' claim faces strongest challenge yet. Nature 590, 19-20 (2021).

55. Greene, T. P. et al. Characterizing transiting exoplanet atmospheres with JWST. Astrophys. J. 817, 17 (2016).

56. Hoffmann, E. H. et al. An advanced modeling study on the impacts and atmospheric implications of multiphase dimethyl sulfide chemistry. Proc. Natl Acad. Sci. USA 113, 11776-11781 (2016).

57. Attard, E. et al. Effects of atmospheric conditions on ice nucleation activity of Pseudomonas. Atmos. Chem. Phys. 12, 10667-10677 (2012).

58. Wagner, W. \& Pruss, A. International equations for the saturation properties of ordinary water substance. Revised according to the International Temperature Scale of 1990. Addendum J. Phys. Chem. Ref. Data 16, 893 (1987). J. Phys. Chem. Ref. Data 22, 783-787 (1993).

59. Wexler, A. S. \& Clegg, S. L. Atmospheric aerosol models for systems including the ions $\mathrm{H}^{+}, \mathrm{NH}_{4}^{+}, \mathrm{Na}^{+}, \mathrm{SO}_{4}{ }^{2-}, \mathrm{NO}_{3}^{-}, \mathrm{Cl}^{-}, \mathrm{Br}^{-}$, and $\mathrm{H}_{2} \mathrm{O}$. J. Geophys. Res. 107, ACH14-1-ACH14-14 (2002).

60. Jia, S. Technical note: Comparison and interconversion of $\mathrm{pH}$ based on different standard states for aerosol acidity characterization. Atmos. Chem. Phys. 18, 11125-11133 (2018) 
61. International Union of Pure and Applied Chemistry IUPAC Compendium of Chemical Terminology 2nd edn (complied by McNaught, A. D. \& Wilkinson, A.) (Blackwell Scientific Publications, 1997); https://doi.org/10.1351/goldbook

62. Lamb, D. \& Verlinde, J. Physics and Chemistry of Clouds (Cambridge Univ. Press, 2011).

63. Camuffo, D. Physics of drop formation and micropore condensation. In Microclimate for Cultural Heritage Conservation, Restoration, and Maintenance of Indoor and Outdoor Monuments (2ed. Camuffo, D.) 165-201 (Elsevier, 2014).

64. Wix, A., Brachert, L., Sinanis, S. \& Schaber, K. A simulation tool for aerosol formation during sulphuric acid absorption in a gas cleaning process. J. Aerosol Sci. 41, 1066-1079 (2010).

65. Gardner, J. A. et al. Measurement of the mass accommodation coefficient of $\mathrm{SO}_{2}$ (g) on water droplets. J. Geophys. Res. 92, 10887-10895 (1987).

66. Schulze-Makuch, D., Grinspoon, D. H., Abbas, O., Irwin, L. N. \& Bullock, M A. A sulfur-based survival strategy for putative phototrophic life in the Venusian atmosphere. Astrobiology 4, 11-18 (2004).

67. Dartnell, L. R. et al. Constraints on a potential aerial biosphere on Venus: I. Cosmic rays. Icarus 257, 396-405 (2015).

68. Winston, P. W. \& Bates, D. H. Saturated solutions for the control of humidity in biological research. Ecology 41, 232-237 (1960).

69. Lee, C. J. D. et al. NaCl-saturated brines are thermodynamically moderate, rather than extreme, microbial habitats. FEMS Microbiol. Rev. 42, 672-693 (2018).

70. Harrison, J. P. et al. Aerobically respiring prokaryotic strains exhibit a broader temperature- $\mathrm{pH}$-salinity space for cell division than anaerobically respiring and fermentative strains. J. R. Soc. Interface 12, 20150658 (2015).

71. Benison, K. C., O’Neill, W. K., Blain, D. \& Hallsworth, J. E. Water activities of acid brine lakes approach the limit for life. Astrobiology https://doi.org/ 10.1089/ast.2020.2334 (2021).

72. Moger-Reischer, R. Z. \& Lennon, J. T. Microbial ageing and longevity. Nat. Rev. Microbiol. 17, 679-690 (2019).

73. Khaleque, H. N., Kaksonen, A. H., Boxall, N. J. \& Watkin, E. L. J. Chloride ion tolerance and pyrite bioleaching capabilities of pure and mixed halotolerant, acidophilic iron- and sulfur-oxidizing cultures. Miner. Eng. 120, 87-93 (2018).

74. Khaleque, H. N. et al. Genome-based classification of two halotolerant extreme acidophiles, Acidihalobacter prosperus V6 (=DSM $14174=$ JCM 32253) and 'Acidihalobacter ferrooxidans' V8 (=DSM $14175=\mathrm{JCM} 32254)$ as two new species, Acidihalobacter aeolianus sp. nov. and Acidihalobacter ferrooxydans sp. nov., respectively. Int. J. Syst. Evol. Microbiol. 69, 1557-1565 (2019).

75. Hallsworth, J. E. et al. Limits of life in $\mathrm{MgCl}_{2}$-containing environments: chaotropicity defines the window. Environ. Microbiol. 9, 801-813 (2007)

76. Stevenson, A., Hamill, P. G., Dijksterhuis, J. \& Hallsworth, J. E. Water-, $\mathrm{pH}$ - and temperature relations of germination for the extreme xerophiles Xeromyces bisporus (FRR 0025), Aspergillus penicillioides (JH06THJ) and Eurotium halophilicum (FRR 2471). Microb. Biotechnol. 10, 330-340 (2017).

77. Rosso, L., Lobry, J. R., Bajard, S. \& Flandrois, J. P. Convenient model to describe the combined effects of temperature and $\mathrm{pH}$ on microbial growth. Appl. Environ. Microbiol. 61, 610-616 (1995).

78. Beale, E. Confidence regions in non-linear estimation. J. R. Stat. Soc. Ser. B 22, 41-88 (1960).

79. Fu, W. \& Zhang, X. Global phosphorus dynamics in terms of phosphine. npj Clim. Atmos. Sci. 3, 51 (2020).

80. Rozenberg, M., Loewenschuss, A. \& Nielsen, C. J. Hydrogen bonding in the sulfuric acid-methanol-water system: A matrix isolation and computational study. J. Phys. Chem. A 119, 2271-2280 (2015).

81. Rozenberg, M., Loewenschuss, A. \& Nielsen, C. J. H-bonding of sulfuric acid with its decomposition products: An infrared matrix isolation and computational study of the $\mathrm{H}_{2} \mathrm{SO}_{4} \cdot \mathrm{H}_{2} \mathrm{O} \cdot \mathrm{SO}_{3}$ complex. J. Phys. Chem. A 120 , 3450-3455 (2016).

82. Stevenson, A. \& Hallsworth, J. E. Water and temperature relations of Actinobacteria. Environ. Microbiol. Rep. 6, 744-755 (2014).

83. Ball, P. \& Hallsworth, J. E. Water structure and chaotropicity: their uses, abuses, and biological implications. Phys. Chem. Chem. Phys. 17, 8297-8305 (2015)

84. Cray, J. A. Chaotropicity: a key factor in product tolerance of biofuel-producing microorganisms. Curr. Opin. Biotechnol. 33, 228-259 (2015)

85. Roy, C. et al. Microbiome and ecology of hot spring-microbialite system on the Trans-Himalayan plateau. Sci. Rep. 10, 5917 (2020).
86. Stevenson, A. et al. Glycerol enhances fungal germination at the water-activity limit for life. Environ. Microbiol. 19, 947-967 (2017).

87. Williams, J. P. \& Hallsworth, J. E. Limits of life in hostile environments: no barriers to biosphere function? Environ. Microbiol. 11, 3292-3308 (2009).

88. Wakisaka, A. \& Matsuura, K. Microheterogeneity of ethanol-water binary mixtures observed at the cluster level. J. Mol. Liq. 129, 25-32 (2006).

89. Zhao, H., Zhanga, Q. \& Du, L. Hydrogen bonding in cyclic complexes of carboxylic acid-sulfuric acid and their atmospheric implications. RSC Adv. 6 , 71733-71743 (2016).

90. Fleming, E. L., Chandra, S., Shoeberl, M. R. \& Barnett, J. J. Monthly Mean Global Climatology of Temperature, Wind, Geopotential Height, and Pressure for 0-120 km NASA Technical Memorandum TM-100697 (NASA, 1988).

91. Warneck, P. \& Williams, J. The Atmospheric Chemist's Companion (Springer, 2012).

92. Bohren, C. F. \& Clothiaux, E. Fundamentals of Atmospheric Radiation (Wiley, 2006).

93. National Oceanic and Atmospheric Administration U.S. Standard Atmosphere 1976 (Governmental Printing Office, 1976).

\section{Acknowledgements}

We are grateful to S. L. Clegg (University of East Anglia, England, UK) for helpful discussions on the use of the E-AIM at low water activity and the provision of some code; C. S. Cockell (University of Edinburgh, Scotland, UK), D. Y. Sorokin (Winogradsky Institute of Microbiology, Russia) and A. Ventosa (University of Seville, Spain) for providing information about thermotolerance of halophiles; M. S. Marley (NASA Ames Research Center, CA, USA) for information on Jupiter and exoplanets; A. Méndez (University of Puerto Rico, Puerto Rico) for inputs relating to analysis of Earth's atmosphere; J. R. Lobry (University of Lyons, France) who helped with use of the cardinal pH model; N. J. Tosca (University of Cambridge, England, UK) for discussions about thermodynamic properties of aqueous sulfuric acid solutions; and E. L. J. Watkin (Curtin University, Australia) who provided information about stress tolerance of Acidihalobacter. J.E.H. was funded by the Biotechnology and Biological Sciences Research Council (BBSRC, United Kingdom) project BBF003471; M.-P.Z. was supported by projects PID2019-104205GB-C21 of Ministry of Science and Innovation and MDM-2017-0737 Unidad de Excelencia 'María de Maeztu'- Centro de Astrobiología (CSIC-INTA) (Spain); and O.V.G. was supported by the Centre of Environmental Biotechnology Project (grant 810280 ) funded by the European Regional Development Fund (ERDF) through the Welsh Government.

\section{Author contributions}

J.E.H., P.B. and M.-P.Z. conceived the study; J.E.H., C.P.M., T.K. and M.-P.Z. designed the approach; all authors obtained and analysed the data (T.K., M.-P.Z., J.E.H. and J.B. for water activity of $\mathrm{H}_{2} \mathrm{SO}_{4}-\mathrm{H}_{2} \mathrm{O}$ mixtures; C.P.M. for the Martian and Jovian atmospheres and relative humidity of the Venusian atmosphere; T.K., C.P.M. and J.E.H. for the Earth case study; T.K., C.P.M., J.E.H., M.-P.Z. and J.B. for quantification of sulfuric acid concentration and water activity of the droplets in Venusian clouds; J.E.H., T.D.D. and O.V.G. for acidity and water-activity limits of life on Earth; M.K.D., P.B. and J.E.H. for activities of sulfuric acid on the cellular system; and J.E.H., T.D.D., C.P.M., M.K.D., M.-P.Z., J.M.-T., T.K., J.B. and P.B. for determination of habitability for Venus's acid clouds); T.K., C.P.M., J.E.H., T.D.D., M.K.D. and M.-P.Z. constructed the displays; J.E.H. produced an initial draft of the manuscript; all authors contributed to writing the final manuscript.

\section{Competing interests}

The authors declare no competing interests.

\section{Additional information}

Supplementary information The online version contains supplementary material available at https://doi.org/10.1038/s41550-021-01391-3.

\section{Correspondence and requests for materials should be addressed to J.E.H.}

Peer review information Nature Astronomy thanks Abel Méndez, Dirk Schulze-Makuch and Nicholas Tosca for their contribution to the peer review of this work.

Reprints and permissions information is available at www.nature.com/reprints.

Publisher's note Springer Nature remains neutral with regard to jurisdictional claims in published maps and institutional affiliations.

(C) The Author(s), under exclusive licence to Springer Nature Limited 2021 